\title{
A Hierarchy of Patient-Reported Outcomes for Meta-Analysis of Knee Osteoarthritis Trials: Empirical Evidence from a Survey of High Impact Journals
}

\author{
Carsten Juhl, ${ }^{1,2}$ Hans Lund, ${ }^{1}$ Ewa M. Roos, ${ }^{1}$ Weiya Zhang, ${ }^{3}$ and Robin Christensen ${ }^{1,4}$ \\ ${ }^{1}$ Research Unit for Musculoskeletal Function and Physiotherapy, Institute of Sports Science and Clinical Biomechanics, \\ University of Southern Denmark, 5230 Odense M, Denmark \\ ${ }^{2}$ Department of Physiotherapy, Copenhagen University Hospital, Gentofte, 2900 Hellerup, Denmark \\ ${ }^{3}$ Academic Rheumatology, University of Nottingham, Nottingham NG5 1PB, UK \\ ${ }^{4}$ Musculoskeletal Statistics Unit, The Parker Institute, Copenhagen University Hospital, Frederiksberg, 2000 Frederiksberg, Denmark \\ Correspondence should be addressed to Robin Christensen, robin.christensen@frh.regionh.dk
}

Received 22 February 2012; Accepted 5 April 2012

Academic Editor: Ruben Burgos-Vargas

Copyright ( $\odot 2012$ Carsten Juhl et al. This is an open access article distributed under the Creative Commons Attribution License, which permits unrestricted use, distribution, and reproduction in any medium, provided the original work is properly cited.

Objectives. To develop a prioritised list based on responsiveness for extracting patient-reported outcomes (PROs) measuring pain and disability for performing meta-analyses in knee osteoarthritis (OA). Methods. A systematic search was conducted in 20 highest impact factor general and rheumatology journals chosen a priori. Eligible studies were randomised controlled trials, using two or more PROs measuring pain and/or disability. Results. A literature search identified 402 publications and 38 trials were included, resulting in 54 randomised comparisons. Thirty-five trials had sufficient data on pain and 15 trials on disability. The WOMAC "pain" and "function" subscales were the most responsive composite scores. The following list was developed. Pain: (1) WOMAC "pain" subscale, (2) pain during activity (VAS), (3) pain during walking (VAS), (4) general knee pain (VAS), (5) pain at rest (VAS), (6) other composite pain scales, and (7) other single item measures. Disability: (1) WOMAC "function" subscale, (2) SF36 "physical function" subscale, (3) SF-36 (Physical composite score), and (4) Other composite disability scores. Conclusions. As choosing the PRO most favourable for the intervention from individual trials can lead to biased estimates, using a prioritised list as developed in this study is recommended to reduce risk of biased selection of PROs in meta-analyses.

\section{Introduction}

Measures for patient-reported outcomes (PROs) are used in most osteoarthritis (OA) trials. Extracting and combining these data is an essential part of any meta-analysis of such trials. At the 3rd Outcome Measures in Rheumatology (OMERACT) Conference in 1996, consensus was reached on three domains - pain, disability, and patient global assessmentas measures that should be reported in future OA trials [1]. This recommendation only specified which constructs to measure, but not which specific instruments to include [2]. In knee OA, pain is typically measured by either the Western Ontario and McMaster Universities Osteoarthritis Index (WOMAC, "pain" subscale) or by different pain scores using a visual analogue scale (VAS). Patient-reported disability is often measured by WOMAC "function" subscale or Short Form 36 (SF-36 "physical functioning” (PF) subscale), but a variety of different PRO measures have been used [3]. Often several PROs are used in trials for estimating change in pain and disability. Simply extracting data on the primary outcome may not be an option in a meta-analysis, as this is often not reported and may not be available for both pain and disability. If the choice of PROs is based on which outcome measure reaches statistical significance, the corresponding meta-analytic estimates are likely to be biased $[4,5]$.

Ideally, the most responsive outcome measure is the best choice for extraction and inclusion in a meta-analysis, provided that it is a valid outcome measure and included in the majority of the trials. Whether the outcome measure is 
truly valid (i.e., it measures the intended change) is a separate consideration based on face, content, construct, and criterion validity [6]. Good validity is a prerequisite for high responsiveness [7]. Responsiveness is quantified as the standardised mean difference (SMD), which is calculated as the difference in the mean change between the intervention and control groups divided by the pooled standard deviation (combining the different groups in any particular trial). The PRO with the largest SMD is considered to be the most responsive [8].

The choice of the most responsive among the potential outcome measures is consistent with the OMERACT filter of truth, discrimination, and feasibility [9]. Combining instruments with different responsiveness can increase heterogeneity in a meta-analysis [10]. Alternatively, various outcomes can be transformed to the most frequently used outcome, using the transformation coefficients from regression analyses $[7,10]$.

In meta-analyses of $\mathrm{OA}$, it is recommended that a hierarchy of PROs should be determined prior to data extraction [11]. In 2006, Jüni et al. suggested a prioritised list for extracting data on pain and disability in patients with knee OA, but the methodology behind the list was not reported [11]. Likewise in a meta-analysis of aquatic exercise for OA, a nonsystematic approach was used to develop an operational hierarchy for OA meta-analyses [12].

1.1. Objectives. The aim of this study was to develop a prioritised list for extracting PROs on pain and disability for metaanalyses, based on an investigation of the responsiveness of $\mathrm{PRO}$ measures in knee OA, and restricted to trials with an anticipated low risk of selective outcome reporting.

\section{Methods}

2.1. Eligibility Criteria. Trials were considered eligible in the current study if they were designed as randomised controlled trials (RCTs) or quasi-randomised trials investigating any type of intervention for patients with knee OA [13]. Trials had to include at least one group with a specified intervention and a control group. In accordance with international consensus regarding the core set of outcome measures for phase III clinical trials in OA [9], the eligible RCTs had to include assessment of at least self-reported pain and/or self-reported disability. Only trials measuring one or both of these constructs with at least two different outcome measures were eligible.

2.2. Information Sources. A systematic literature search was conducted in MEDLINE in December 2009 by searching PubMed in order to identify RCTs of interventions aimed at reducing pain and disability in patients with knee OA. In order to identify RCTs with a low risk of selective outcome reporting, the search was restricted to RCTs published later than the year of 2002. The revised CONSORT Statement was published in 2001, and the reporting of RCTs was expected to improve from 2002 [14].
2.3. Literature Search. The search terms for identifying knee OA were "osteoarthritis, knee" [MeSH] OR ("osteoarthritis" [TIAB] AND "knee" [TIAB]). The search was restricted to the ten highest impact factor general and internal medicine journals (previous 5 years) as well as the ten highest impact factor rheumatology journals (previous 5 years) (determined by Journal Citation Reports, http://isiwebofknowledge.com. 6 November 2009): The New England Journal of The Medicine; Journal of the American Medical Association; The Lancet; Annals of Internal Medicine; British Medical Journal; PLoS Medicine; Annual Review of Medicine; Archives of Internal Medicine; Canadian Medical Association Journal; Medicine, Arthritis and Rheumatism; Annals of Rheumatic Diseases; Nature Clinical Practice Rheumatology; Arthritis Research \& Therapy; Seminars in Arthritis and Rheumatism; Osteoarthritis and Cartilage, Rheumatology (Oxford); Current Opinion in Rheumatology; Journal of Rheumatology; and Best Practice and Research in Clinical Rheumatology. Targeting journals with a high impact factor has previously been suggested as a good strategy for identifying journal articles with high methodological quality [15].

2.4. Study Selection. Two members of the study team (C. Juhl, H. Lund) independently scrutinised titles and abstracts of all identified publications. The full text of any article was obtained if it was judged eligible by at least one of the reviewers. The two reviewers then evaluated eligibility based on the full text of all the retrieved papers, and consensus on inclusion was reached by discussion.

2.5. Data Collections Process and Data Items. Study identification (author, year) and outcome measures were extracted using a customised data extraction form. For each outcome measure, the number of participants in the intervention and the control groups and the mean change and standard deviation (SD) were extracted in order to calculate the SMD. When SD was not available in an explicit format, it was estimated from the standard error (SE), confidence interval, the $P$ value, the interquartile range, or other methods as recommended by the Cochrane Collaboration [16].

2.6. Risk of Bias in Individual Studies. Selective outcome reporting has been defined as choosing a subset of the original outcomes on the basis of the results $[1,2]$. Two members of the study team (C. Juhl, H. Lund) assessed the risk of selective outcome reporting, indexed according to whether the trials had been classified as "adequate," "unclear," or "inadequate" in accordance with the Cochrane Handbook for Systematic Reviews of Interventions 5.1.0 [16]. Selective outcome reporting was deemed as follows.

(i) Adequate, if a protocol (published or from ClinicalTrials gov or other databases) was available and all PROs were sufficiently reported for extracting data for estimating SMD.

(ii) Unclear, if a protocol was not available (published or from ClinicalTrials gov or other databases). 


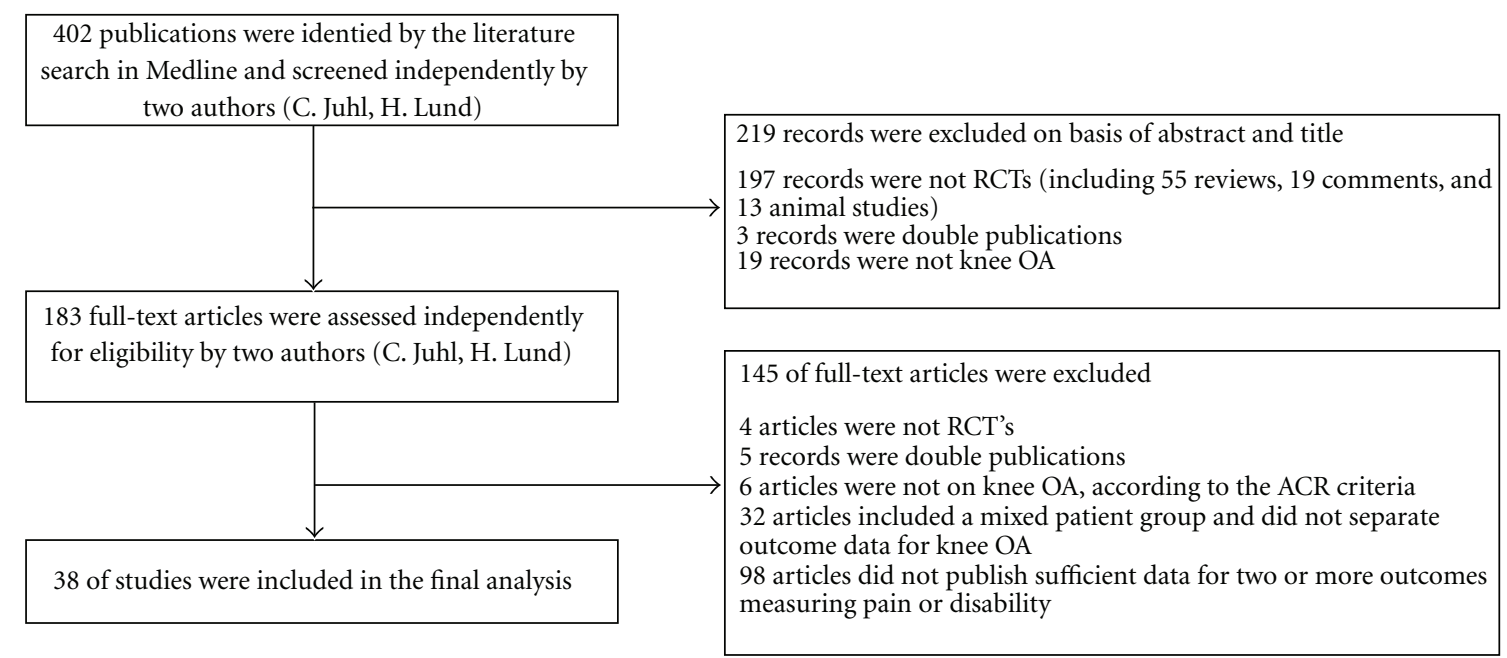

FIGURE 1: Flowchart: identification of studies.

(iii) Inadequate, if some or all PROs were insufficiently reported for extracting data for estimating SMD (evaluated by checking the protocol from earlier publications, trial registers, or described in the published trial).

2.7. Summary Measures. The effect size was calculated as the SMD to allow comparison of the various PROs. The SMD was estimated as the difference in mean change between the intervention and control groups divided by the pooled $\mathrm{SD}$. The pooled SD was estimated from $\mathrm{SD}_{\text {pooled }}{ }^{2}=\left(\mathrm{SD}_{I}{ }^{2} \times\right.$ $\left.\left[N_{I}-1\right]+\mathrm{SD}_{C}{ }^{2} \times\left[N_{C}-1\right]\right) /\left(N_{I}+N_{C}-2\right)$, where $N_{I}$ and $\mathrm{SD}_{I}$ represent the number of patients and the $\mathrm{SD}$ in the intervention group, respectively. The SMD was used to represent the responsiveness; the higher the SMD, the more responsive the measure. This approach is only valid to rank PROs within a particular study, as different studies obviously measure different therapeutic interventions. Data from an intention to treat (ITT) analysis was preferred for calculating the SMD. When several intervention groups were compared with a control group, the number of control patients was divided equally into the appropriate number of groups when estimating the SMD.

2.8. Synthesis of Results. The responsiveness estimated as SMD of the PROs in each of the included trials (or subgroups when more interventions were compared with the control) was ranked according to responsiveness for pain and disability separately. The PRO used to measure the effect of an intervention in any individual trial with the highest responsiveness was ranked 1 , the second most responsive was ranked 2, and so on. The mean rank was then used to estimate the responsiveness across the trials, and a low mean rank (close to 1) indicated that this PRO was often the most responsive PRO used. The PROs used in at least 5 trials were then listed according to the lowest mean rank of the SMD. However, composite item scales with established validity would rate higher than single item scales.
2.9. Risk of Bias across Studies. A sensitivity analysis was performed evaluating the impact of different systematic approaches to data extraction of PROs in a meta-analysis. The pooled mean across trials using the developed list from the current study was compared with lists based on (1) the most favourable outcome from each of the individual trials, (2) the most frequently used PROs, and (3) the most responsive of the PROs. Inconsistency in means between trials was evaluated using the $I^{2}$ index [17]. A random effect model was used for pooling the trials. All analyses were performed at the study level using the meta-analysis software "Comprehensive Meta Analysis" Version 2, Biostat. Inc., and were based on published data only.

2.10. Additional Analysis. In order to assess the robustness of the developed list, subgroup analyses stratifying the available trials according to risk of selective outcome bias were applied, and a list based on trials with no risk of selective outcome bias was compared with the list based on all included trials. Stratified analyses were performed based on whether the included trials were published in the general/internal medicine journals or in the rheumatology journals. Secondly, sensitivity analyses were performed stratifying trials according to the intervention; injection in the knee joint, oral medication and other interventions (tai chi, lateral wedge shoes, etc.). Outcomes used at least 5 times were ranked based on the mean rank and the lists from these subgroups were compared to the list based of all included trials.

\section{Results}

3.1. Study Selection. Through the search strategy, 402 publications were identified, as presented in the flowchart (Figure 1). Titles and abstracts of the publications were checked independently by two reviewers (C. Juhl, H. Lund). One hundred and eighty three trials were identified as potentially eligible by at least one of the reviewers and subsequently examined independently in full text by two 
reviewers (C. Juhl, H. Lund). Consensus was reached by discussion and resulted in 38 trials that fulfilled the eligibility criteria and were included in the analysis [18-55] (Table 1).

3.2. Study Characteristics. Pain was evaluated with more than one PRO in 35 trials [18-21, 23-42, 45-55], and disability was evaluated in 15 trials $[18,19,21,22,25,26,29,31,38$, $40,43,44,46,53,54]$. More than one intervention group was compared in 14 trials measuring pain $[19,24,25,27-29,34-$ $36,41,42,48-50,55]$ and in 4 trials measuring disability $[22,25,29,40]$. Different specific questions were asked when VAS scores were used for measuring pain. The VAS scores were classified as pain during activity (e.g., stepping, daily activities, etc.), pain during walking, pain at rest, pain at night, and general knee pain. WOMAC was used both in a VAS version using a $100 \mathrm{~mm}$ scale and in a Likert version using a 1-5 scale, and these were treated as different outcomes in the initial analysis. Pain during activity and pain during walking were measured by either a VAS score or a numeric rating scale (NRS) score, and these PROs were analysed separately. Then eighteen different PROs were used for measuring pain and seven PROs for measuring disability in the included trials.

3.3. Risk of Bias within Studies. Only 12 out of the 38 included trials were registered in ClinicalTrial.gov. Six of these trials were classified as "adequate" and six as "inadequate". Two trials reported PROs not declared in the protocol, one did not report all PROs from the published protocol, one did not report all time points, and two protocols did not report PROs at all in the published protocol (Table 1).

3.4. Results of Individual Studies. The most frequently used PROs for measuring pain were the WOMAC "pain" subscale (in either the Likert scale or VAS format) used in 27 of the included trials and for disability the most frequently used PRO was the WOMAC "function" subscale (in either the Likert scale or VAS format) used in all 15 trials (Table 2).

The most responsive PRO measure for pain was "pain during activity" using a VAS with a mean rank of 1.4. It was used when comparing the effect of an intervention in 12 trials (comparing 18 interventions with controls), and it was the most responsive outcome in 14 of these 18 substudies. The most responsive composite score for pain was the WOMAC "pain" subscale using a Likert scale with a mean rank of 1.8. It was the most responsive outcome in 7 out of 20 substudies and the most responsive composite score in 19 out of 20 substudies. The most responsive PRO on disability was WOMAC "function" subscale using a $100 \mathrm{~mm}$ scale with a mean rank of 1.4. It was the most responsive outcome in 7 out of 13 substudies.

3.5. Synthesis of Results. According to the Food and Drug Administration (FDA), a single item may be reasonable for concepts such as pain severity, if it is a reliable and valid measure, but not for general concepts such as disability [56]. Different single item VAS scores were actually measuring different items, as they were based on different questions. A list based on the responsiveness of the PROs was constructed, placing the most responsive of the PROs as first choice for extracting data for meta-analyses, and PROs based on a single item being downgraded as they were derived from different instruments, whose validity and reliability had not been established. The final prioritised list for preferred PROs to extract when performing meta-analyses is presented in Table 3.

3.6. Risk of Bias across Studies. The likelihood of overestimating the pooled effect in meta-analyses by choosing the most favourable PROs for the intervention in each trial is illustrated in Figure 2. Compared with the developed list, consistently choosing the most favourable PROs overestimated the effect size for pain with SMD $=0.14(95 \%$ CI $0.02,0.26)$ and for disability with SMD $=0.09$ (95\% CI -0.03 , 0.21 ). The differences between (1) the developed list, (2) the most frequently used PROs, and (3) the most responsive of the PROs were smaller. The list developed in this study seems to be a conservative but robust approach for extracting PROs when planning a meta-analysis.

3.7. Additional Analysis. The subgroup analysis stratifying the available trials according to risk of selective outcome bias was applied, and a list based on trials with a low risk of selective outcome reporting was developed (i.e., trials with a published protocol and evaluated as "adequate" in the risk of bias assessment). All 6 trials with a low risk of selective outcome reporting had measured pain, but only 3 had measured disability. Even though some outcomes were missing in the list based on these 6 trials with low risk of selective outcome reporting bias compared with the list based on all the included 38 trials, the differences between the two lists were very small (data not shown).

When stratifying the included trials according to the interventions; injection in the knee joint, oral medication and other interventions (tai chi, lateral wedge shoes etc.) no differences in the responsiveness of the most frequently used outcomes (a least 5 times) were found between the "injection subgroup", the "other interventions subgroup" and all the included trials. In the "oral medication subgroup" the five most responsive outcomes (used a least 5 times) were "Pain walking", "Pain global", WOMAC (100 mm scale), "Pain at rest" and WOMAC (likert scale). When compared this to the list of outcomes from all the included trials "Pain activity" and SF-36 is missing as they were not used as frequently in the "oral medication subgroup" and the rank of the last three later were WOMAC (likert scale), WOMAC (100 mm scale) and "Pain at rest" but the responsiveness for these three outcomes was more or less the same. No differences in sensitivity analysis based on intervention were seen in the disability list. Other sensitivity analyses based on whether the included trials were published in the general/internal medicine journals or in the rheumatology journals showed no differences between lists based on mean rank in these subgroups and the list of all trials neither for the analysis of disability nor for pain. 
TABLE 1: Characteristics of included studies.

\begin{tabular}{|c|c|c|c|c|c|c|c|c|c|}
\hline Author & Journal & Comparison & $\begin{array}{l}\text { Number of } \\
\text { randomised } \\
\text { patients }\end{array}$ & Construct & $\begin{array}{l}\text { Selective } \\
\text { outcome } \\
\text { reporting }\end{array}$ & Outcome & $\begin{array}{l}\text { Number of } \\
\text { patients }\end{array}$ & $\begin{array}{l}\text { Standard mean } \\
\text { differences } \\
(\text { SMD) } \\
\text { measured as } \\
\text { Cohens'd (SE) }\end{array}$ & $\begin{array}{c}\text { Rank of } \\
\text { responsiveness } \\
\text { measured as } \\
\text { SMD }\end{array}$ \\
\hline \multirow[t]{5}{*}{$\begin{array}{l}\text { Altman } \\
\text { et al. } \\
\text { (Flexx) } \\
2009\end{array}$} & $\begin{array}{l}\text { Semin } \\
\text { Arthritis } \\
\text { Rheum. }\end{array}$ & $\begin{array}{l}\text { Sodium } \\
\text { hyaluronate versus } \\
\text { saline }\end{array}$ & 588 & Pain & Unclear & WOMAC & $291 / 295$ & $0.143(0.083)$ & 2 \\
\hline & & & & & & $\begin{array}{c}\text { VAS } \\
\text { (walking) }\end{array}$ & $285 / 295$ & $0.259(0.083)$ & 1 \\
\hline & & & & & & WOMAC & $291 / 295$ & $0.194(0.083)$ & 1 \\
\hline & & & & Disability & & $\begin{array}{l}\text { SF-36 } \\
\text { (PCS) }\end{array}$ & $291 / 295$ & $0.191(0.083)$ & 2 \\
\hline & & & & & & $\begin{array}{l}\text { SF-36 } \\
(\mathrm{PF})\end{array}$ & $291 / 295$ & $0.170(0.083)$ & 3 \\
\hline \multirow[t]{8}{*}{$\begin{array}{l}\text { Baltzer } \\
\text { et al. } \\
2009\end{array}$} & $\begin{array}{l}\text { Osteoarthritis } \\
\text { and Cartilage }\end{array}$ & $\begin{array}{l}\text { Autologous } \\
\text { conditioned serum } \\
\text { (ACS) versus saline }\end{array}$ & 241 & Pain & Unclear & WOMAC & $134 / 107$ & $0.703(0.166)$ & 2 \\
\hline & & & & & & $\begin{array}{c}\text { VAS } \\
\text { (activity) }\end{array}$ & $134 / 107$ & $0.937(0.169)$ & 1 \\
\hline & & & & Disability & & WOMAC & $134 / 107$ & $0.699(0.166)$ & 1 \\
\hline & & & & & & $\begin{array}{l}\text { SF-8 } \\
\text { (PCS) }\end{array}$ & $134 / 107$ & $0.682(0.166)$ & 2 \\
\hline & & $\begin{array}{l}\text { Hyaluronan versus } \\
\text { saline }\end{array}$ & 242 & Pain & Unclear & WOMAC & $135 / 107$ & $0.050(0.162)$ & 1 \\
\hline & & & & & & $\begin{array}{c}\text { VAS } \\
\text { (activity) }\end{array}$ & $135 / 107$ & $0.035(0.162)$ & 2 \\
\hline & & & & Disability & & WOMAC & $135 / 107$ & $0.078(0.162)$ & 2 \\
\hline & & & & & & $\begin{array}{l}\text { SF-8 } \\
\text { (PCS) }\end{array}$ & $135 / 107$ & $0.146(0.162)$ & 1 \\
\hline \multirow[t]{2}{*}{$\begin{array}{l}\text { Barthel } \\
\text { et al. } \\
2009\end{array}$} & $\begin{array}{l}\text { Semin Arthri- } \\
\text { tis Rheum. }\end{array}$ & $\begin{array}{l}\text { Diclofenac versus } \\
\text { vehicle }\end{array}$ & 492 & Pain & Unclear & WOMAC & $254 / 238$ & $0.234(0.091)$ & 2 \\
\hline & & & & & & $\begin{array}{c}\text { VAS } \\
\text { (activity) }\end{array}$ & $254 / 238$ & $0.281(0.091)$ & 1 \\
\hline \multirow[t]{6}{*}{$\begin{array}{l}\text { Bennell } \\
\text { et al. } \\
2005\end{array}$} & $\begin{array}{l}\text { Ann. Rheum. } \\
\text { Dis. }\end{array}$ & $\begin{array}{l}\text { Multimodal } \\
\text { physiotherapy } \\
\text { programme versus } \\
\text { placebo }\end{array}$ & 140 & Pain & Unclear & WOMAC & $73 / 67$ & $0.122(0.169)$ & 3 \\
\hline & & & & & & $\begin{array}{c}\text { VAS } \\
\text { (activity) }\end{array}$ & $73 / 67$ & $0.223(0.170)$ & 1 \\
\hline & & & & & & $\begin{array}{l}\text { SF-36 } \\
(\mathrm{BP})\end{array}$ & $73 / 67$ & $0.071(0.169)$ & 4 \\
\hline & & & & & & $\begin{array}{l}\text { VAS } \\
\text { (knee) }\end{array}$ & $73 / 67$ & $0.216(0.170)$ & 2 \\
\hline & & & & Disability & & WOMAC & $73 / 67$ & $0.085(0.169)$ & 2 \\
\hline & & & & & & $\begin{array}{c}\text { SF-36 } \\
(\mathrm{PF})\end{array}$ & $73 / 67$ & $0.236(0.170)$ & 1 \\
\hline \multirow[t]{4}{*}{$\begin{array}{l}\text { Berman } \\
\text { et al. } \\
2004\end{array}$} & $\begin{array}{l}\text { Ann. Intern. } \\
\text { Med. }\end{array}$ & $\begin{array}{l}\text { Acupuncture } \\
\text { versus } \\
\text { sham acupuncture }\end{array}$ & 381 & Disability & Unclear & WOMAC & $142 / 141$ & $0.201(0.146)$ & 1 \\
\hline & & & & & & $\begin{array}{l}\text { SF-36 } \\
\text { (PCS) }\end{array}$ & $142 / 141$ & $0.134(0.146)$ & 2 \\
\hline & & $\begin{array}{l}\text { Education versus } \\
\text { sham acupuncture }\end{array}$ & 380 & Disability & Unclear & WOMAC & $141 / 108$ & $-0.244(0.154)$ & 2 \\
\hline & & & & & & $\begin{array}{l}\text { SF-36 } \\
\text { (PCS) }\end{array}$ & $141 / 108$ & $-0.255(0.154)$ & 1 \\
\hline
\end{tabular}


TABle 1: Continued.

\begin{tabular}{|c|c|c|c|c|c|c|c|c|c|}
\hline Author & Journal & Comparison & $\begin{array}{c}\text { Number of } \\
\text { randomised } \\
\text { patients }\end{array}$ & Construct & $\begin{array}{l}\text { Selective } \\
\text { outcome } \\
\text { reporting }\end{array}$ & Outcome & $\begin{array}{l}\text { Number of } \\
\text { patients }\end{array}$ & $\begin{array}{l}\text { Standard mean } \\
\text { differences } \\
\text { (SMD) } \\
\text { measured as } \\
\text { Cohens'd (SE) }\end{array}$ & $\begin{array}{l}\text { Rank of } \\
\text { responsiveness } \\
\text { measured as } \\
\text { SMD }\end{array}$ \\
\hline \multirow[t]{2}{*}{$\begin{array}{l}\text { Burch } \\
\text { et al. } \\
2008\end{array}$} & $\begin{array}{l}\text { Osteoarthritis } \\
\text { and Cartilage }\end{array}$ & $\begin{array}{l}\text { Pattern stimulation } \\
\text { versus control } \\
\text { (TENS) }\end{array}$ & 116 & Pain & Unclear & WOMAC & $52 / 53$ & $0.698(0.201)$ & 1 \\
\hline & & & & & & $\begin{array}{c}\text { VAS } \\
\text { (global) }\end{array}$ & $52 / 53$ & $0.231(0.195)$ & 2 \\
\hline \multirow[t]{4}{*}{$\begin{array}{l}\text { Case } \\
\text { et al. } \\
2003\end{array}$} & $\begin{array}{l}\text { Ann. Intern. } \\
\text { Med. }\end{array}$ & $\begin{array}{l}\text { Diclofena versus } \\
\text { placebo }\end{array}$ & 53 & Pain & Unclear & WOMAC & $25 / 28$ & $0.446(0.338)$ & 1 \\
\hline & & & & & & Lequesne & $25 / 28$ & $0.190(0.334)$ & 2 \\
\hline & & $\begin{array}{l}\text { Acetaminophen } \\
\text { versus placebo }\end{array}$ & 57 & Pain & Unclear & WOMAC & $29 / 28$ & $0.096(0.326)$ & 2 \\
\hline & & & & & & Lequesne & $29 / 28$ & $0.217(0.326)$ & 1 \\
\hline \multirow[t]{16}{*}{$\begin{array}{l}\text { Clegg } \\
\text { et al. } \\
2006\end{array}$} & $\begin{array}{l}\text { N. Engl. J. } \\
\text { Med. }\end{array}$ & $\begin{array}{l}\text { Glucosamine }+ \\
\text { chondroitin versus } \\
\text { placebo }\end{array}$ & 630 & Pain & Inadequate & WOMAC & $317 / 313$ & $0.127(0.126)$ & 2 \\
\hline & & & & & & HAQ & $317 / 313$ & $0.146(0.126)$ & 1 \\
\hline & & & & Disability & & WOMAC & $317 / 313$ & $0.137(0.126)$ & 1 \\
\hline & & & & & & HAQ & $317 / 313$ & $0.104(0.126)$ & 2 \\
\hline & & $\begin{array}{l}\text { Glucosamine } \\
\text { versus placebo }\end{array}$ & 630 & Pain & Inadequate & WOMAC & $317 / 313$ & $-0.028(0.126)$ & 1 \\
\hline & & & & & & HAQ & $317 / 313$ & $-0.021(0.126)$ & 2 \\
\hline & & & & Disability & & WOMAC & $317 / 313$ & $-0.013(0.126)$ & 2 \\
\hline & & & & & & HAQ & $317 / 313$ & $0.056(0.126)$ & 1 \\
\hline & & $\begin{array}{l}\text { Chondroitin versus } \\
\text { placebo }\end{array}$ & 631 & Pain & Inadequate & WOMAC & $318 / 313$ & $-0.020(0.126)$ & 2 \\
\hline & & & & & & HAQ & $318 / 313$ & $-0.046(0.126)$ & 1 \\
\hline & & & & Disability & & WOMAC & $318 / 313$ & $0.023(0.126)$ & 2 \\
\hline & & & & & & HAQ & $318 / 313$ & $0.029(0.126)$ & 1 \\
\hline & & $\begin{array}{l}\text { Celecoxib versus } \\
\text { placebo }\end{array}$ & 631 & Pain & Inadequate & WOMAC & $318 / 313$ & $0.132(0.126)$ & 1 \\
\hline & & & & & & HAQ & $318 / 313$ & $0.130(0.126)$ & 2 \\
\hline & & & & Disability & & WOMAC & $318 / 313$ & $0.179(0.126)$ & 1 \\
\hline & & & & & & HAQ & $318 / 313$ & $0.114(0.126)$ & 2 \\
\hline \multirow[t]{5}{*}{$\begin{array}{l}\text { Forestier } \\
\text { et al. } \\
2009\end{array}$} & $\begin{array}{l}\text { Ann. Rheum. } \\
\text { Dis. }\end{array}$ & $\begin{array}{l}\text { Spa therapy versus } \\
\text { control }\end{array}$ & 451 & Pain & Adequate & $\begin{array}{l}\text { VAS } \\
\text { (knee) }\end{array}$ & $193 / 186$ & $0.310(0.103)$ & 1 \\
\hline & & & & & & $\begin{array}{l}\text { SF-36 } \\
(\mathrm{BP})\end{array}$ & $194 / 185$ & $0.190(0.103)$ & 2 \\
\hline & & & & Disability & & WOMAC & $179 / 172$ & $0.366(0.108)$ & 1 \\
\hline & & & & & & $\begin{array}{l}\text { SF-36 } \\
\text { (PCS) }\end{array}$ & $190 / 177$ & $0.153(0.105)$ & 2 \\
\hline & & & & & & $\begin{array}{l}\text { SF-36 } \\
\text { (PF) }\end{array}$ & $195 / 186$ & $0.070(0.103)$ & 3 \\
\hline \multirow[t]{3}{*}{$\begin{array}{l}\text { Geba } \\
\text { et al. } \\
2002\end{array}$} & JAMA & $\begin{array}{l}\text { Rofecoxib } 25 \mathrm{mg} / \mathrm{d} \\
\text { versus } \\
\text { acetaminophen }\end{array}$ & 189 & Pain & Inadequate & $\begin{array}{c}\text { VAS } \\
\text { (walking) }\end{array}$ & $95 / 94$ & $0.467(0.208)$ & 1 \\
\hline & & & & & & $\begin{array}{c}\text { VAS } \\
\text { (night) }\end{array}$ & $95 / 94$ & $0.394(0.207)$ & 3 \\
\hline & & & & & & VAS (rest) & $95 / 94$ & $0.411(0.206)$ & 2 \\
\hline
\end{tabular}


Table 1: Continued.

\begin{tabular}{|c|c|c|c|c|c|c|c|c|c|}
\hline Author & Journal & Comparison & $\begin{array}{l}\text { Number of } \\
\text { randomised } \\
\text { patients }\end{array}$ & Construct & $\begin{array}{l}\text { Selective } \\
\text { outcome } \\
\text { reporting }\end{array}$ & Outcome & $\begin{array}{c}\text { Number of } \\
\text { patients }\end{array}$ & $\begin{array}{l}\text { Standard mean } \\
\text { differences } \\
(\mathrm{SMD}) \\
\text { measured as } \\
\text { Cohens'd (SE) }\end{array}$ & $\begin{array}{c}\text { Rank of } \\
\text { responsiveness } \\
\text { measured as } \\
\text { SMD }\end{array}$ \\
\hline & & $\begin{array}{l}\text { Rofecixib } \\
12.5 \mathrm{mg} / \mathrm{d} \text { versus } \\
\text { acetaminophen }\end{array}$ & 190 & Pain & Inadequate & $\begin{array}{c}\text { VAS } \\
\text { (walking) }\end{array}$ & $96 / 94$ & $0.191(0.206)$ & 1 \\
\hline & & & & & & $\begin{array}{c}\text { VAS } \\
\text { (night) }\end{array}$ & $96 / 94$ & $0.069(0.206)$ & 3 \\
\hline & & & & & & $\begin{array}{l}\text { VAS } \\
\text { (rest) }\end{array}$ & $96 / 94$ & $0.135(0.206)$ & 2 \\
\hline & & $\begin{array}{l}\text { Celecoxib ver- } \\
\text { sus Acetaminophen }\end{array}$ & 191 & Pain & Inadequate & $\begin{array}{c}\text { VAS } \\
\text { (walking) }\end{array}$ & $97 / 94$ & $0.233(0.206)$ & 1 \\
\hline & & & & & & $\begin{array}{l}\text { VAS } \\
\text { (night) }\end{array}$ & $97 / 94$ & $-0.043(0.206)$ & 3 \\
\hline & & & & & & $\begin{array}{l}\text { VAS } \\
\text { (rest) }\end{array}$ & $97 / 94$ & $0.074(0.206)$ & 2 \\
\hline \multirow{4}{*}{$\begin{array}{l}\text { Gibofsky } \\
\text { et al. } \\
2003\end{array}$} & $\begin{array}{l}\text { Arthritis, } \\
\text { Rheum. }\end{array}$ & $\begin{array}{l}\text { Rofecoxib versus } \\
\text { placebo }\end{array}$ & 285 & Pain & Unclear & WOMAC & $189 / 96$ & $0.489(0.163)$ & 1 \\
\hline & & & & & & $\begin{array}{c}\text { VAS } \\
\text { (walking) }\end{array}$ & $189 / 96$ & $0.366(0.162)$ & 2 \\
\hline & & $\begin{array}{l}\text { Celecoxib versus } \\
\text { placebo }\end{array}$ & 286 & Pain & Unclear & WOMAC & $190 / 96$ & $0.514(0.163)$ & 1 \\
\hline & & & & & & $\begin{array}{c}\text { VAS } \\
\text { (walking) }\end{array}$ & $190 / 96$ & $0.451(0.163)$ & 2 \\
\hline \multirow[t]{12}{*}{$\begin{array}{l}\text { Hinman } \\
\text { et al. } \\
2003\end{array}$} & $\mathrm{BMJ}$ & $\begin{array}{l}\text { Tappening versus } \\
\text { placebo }\end{array}$ & 58 & Pain & Unclear & WOMAC & $29 / 29$ & $0.160(0.318)$ & 4 \\
\hline & & & & & & $\begin{array}{c}\text { NRS } \\
\text { (activity) }\end{array}$ & $29 / 29$ & $0.802(0.329)$ & 1 \\
\hline & & & & & & $\begin{array}{c}\text { NRS } \\
\text { (walking) }\end{array}$ & $29 / 29$ & $0.795(0.329)$ & 2 \\
\hline & & & & & & $\begin{array}{l}\text { SF-36 } \\
\text { (BP) }\end{array}$ & $29 / 29$ & $0.308(0.320)$ & 3 \\
\hline & & & & Disability & & WOMAC & $29 / 29$ & $1.019(0.340)$ & 1 \\
\hline & & & & & & $\begin{array}{l}\text { SF-36 } \\
(\mathrm{PF})\end{array}$ & $29 / 29$ & $0.247(0.323)$ & 2 \\
\hline & & $\begin{array}{l}\text { Control tape versus } \\
\text { placebo }\end{array}$ & 58 & Pain & Unclear & WOMAC & $29 / 29$ & $0.649(0.329)$ & 1 \\
\hline & & & & & & $\begin{array}{c}\text { NRS } \\
\text { (activity) }\end{array}$ & $29 / 29$ & $0.376(0.329)$ & 4 \\
\hline & & & & & & $\begin{array}{c}\text { NRS } \\
\text { (walking) }\end{array}$ & $29 / 29$ & $0.649(0.329)$ & 1 \\
\hline & & & & & & $\begin{array}{l}\text { SF-36 } \\
\text { (BP) }\end{array}$ & $29 / 29$ & $0.431(0.325)$ & 3 \\
\hline & & & & Disability & & WOMAC & $29 / 29$ & $0.640(0.329)$ & 1 \\
\hline & & & & & & $\begin{array}{l}\text { SF-36 } \\
(\mathrm{PF})\end{array}$ & $29 / 29$ & $0.230(0.323)$ & 2 \\
\hline \multirow[t]{2}{*}{$\begin{array}{l}\text { Hughes } \\
\text { and } \\
\text { Carr } 2002\end{array}$} & Rheumatology & $\begin{array}{l}\text { Glucosamine } \\
\text { sulphate versus } \\
\text { placebo }\end{array}$ & 80 & Pain & Unclear & WOMAC & $39 / 39$ & $-0.066(0.227)$ & 3 \\
\hline & & & & & & $\begin{array}{c}\text { VAS } \\
\text { (activity) }\end{array}$ & $39 / 39$ & $-0.020(0.226)$ & 5 \\
\hline
\end{tabular}


TABle 1: Continued.

\begin{tabular}{|c|c|c|c|c|c|c|c|c|c|}
\hline Author & Journal & Comparison & $\begin{array}{l}\text { Number of } \\
\text { randomised } \\
\text { patients }\end{array}$ & Construct & $\begin{array}{l}\text { Selective } \\
\text { outcome } \\
\text { reporting }\end{array}$ & Outcome & $\begin{array}{l}\text { Number of } \\
\text { patients }\end{array}$ & $\begin{array}{c}\text { Standard mean } \\
\text { differences } \\
\text { (SMD) } \\
\text { measured as } \\
\text { Cohens'd (SE) }\end{array}$ & $\begin{array}{c}\text { Rank of } \\
\text { responsiveness } \\
\text { measured as } \\
\text { SMD }\end{array}$ \\
\hline & & & & & & $\begin{array}{l}\text { VAS } \\
\text { (knee) }\end{array}$ & $39 / 39$ & $-0.032(0.226)$ & 4 \\
\hline & & & & & & $\begin{array}{l}\text { VAS } \\
\text { (rest) }\end{array}$ & $39 / 39$ & $0.323(0.228)$ & 1 \\
\hline & & & & & & $\begin{array}{l}\text { McGill } \\
\text { (sense) }\end{array}$ & $39 / 39$ & $0.221(0.227)$ & 2 \\
\hline \multirow[t]{5}{*}{$\begin{array}{l}\text { Kirkley } \\
\text { et al. } \\
2008\end{array}$} & $\begin{array}{l}\text { N. Engl. J. } \\
\text { Med. }\end{array}$ & $\begin{array}{l}\text { Arthroscopic } \\
\text { surgery } \\
\text { versus } \\
\text { placebo }\end{array}$ & 188 & Pain & Adequate & WOMAC & $88 / 80$ & $0.316(0.155)$ & 1 \\
\hline & & & & & & ASES & $88 / 80$ & $-0.073(0.155)$ & 2 \\
\hline & & & & Disability & & WOMAC & $88 / 80$ & $0.259(0.155)$ & 1 \\
\hline & & & & & & ASES & $88 / 80$ & $0.209(0.155)$ & 2 \\
\hline & & & & & & $\begin{array}{l}\text { SF-36 } \\
\text { (PCS) }\end{array}$ & $88 / 80$ & $-0.009(0.155)$ & 3 \\
\hline \multirow[t]{2}{*}{$\begin{array}{l}\text { Kitay } \\
\text { et al. } \\
2009\end{array}$} & $\begin{array}{l}\text { Osteoarthritis } \\
\text { and Cartilage }\end{array}$ & $\begin{array}{l}\text { Vibrations, } \\
\text { CPM, and } \\
\text { heat versus } \\
\text { sham device }\end{array}$ & 71 & Pain & Adequate & WOMAC & $24 / 32$ & $0.984(0.284)$ & 1 \\
\hline & & & & & & $\begin{array}{l}\text { VAS } \\
\text { (knee) }\end{array}$ & $24 / 32$ & $0.984(0.284)$ & 1 \\
\hline \multirow[t]{2}{*}{$\begin{array}{l}\text { Mazières } \\
\text { et al. } \\
2007\end{array}$} & $\begin{array}{l}\text { Ann. Rheum. } \\
\text { Dis. }\end{array}$ & $\begin{array}{l}\text { Chondroitin } \\
\text { sulphate } \\
\text { versus } \\
\text { placebo }\end{array}$ & 307 & Pain & Unclear & $\begin{array}{c}\text { VAS } \\
\text { (activity) }\end{array}$ & $139 / 140$ & $0.260(0.120)$ & 1 \\
\hline & & & & & & $\begin{array}{l}\text { VAS } \\
\text { (rest) }\end{array}$ & $139 / 140$ & $0.092(0.120)$ & 2 \\
\hline \multirow[t]{2}{*}{$\begin{array}{l}\text { McCarthy } \\
\text { et al. } \\
2004\end{array}$} & Rheumatology & $\begin{array}{l}\text { Class-based } \\
\text { versus } \\
\text { home-based } \\
\text { exercise }\end{array}$ & 214 & Pain & Unclear & WOMAC & $111 / 103$ & $0.321(0.138)$ & 2 \\
\hline & & & & & & $\begin{array}{c}\text { VAS } \\
\text { (walking) }\end{array}$ & $111 / 103$ & $0.836(0.143)$ & 1 \\
\hline \multirow[t]{8}{*}{$\begin{array}{l}\text { Moseley } \\
\text { et al. } \\
2002\end{array}$} & $\begin{array}{l}\text { N. Engl. J. } \\
\text { Med. }\end{array}$ & $\begin{array}{l}\text { Arthroscopic } \\
\text { debridement } \\
\text { versus } \\
\text { placebo }\end{array}$ & 119 & Pain & Unclear & $\begin{array}{c}\text { Knee } \\
\text { specific }\end{array}$ & & & \\
\hline & & & & & & Pain scale & $53 / 55$ & $0.010(0.232)$ & 3 \\
\hline & & & & & & $\begin{array}{l}\text { AIMS } \\
\text { (pain) }\end{array}$ & $53 / 55$ & $-0.072(0.224)$ & 2 \\
\hline & & & & & & $\begin{array}{l}\text { SF-36 } \\
(\mathrm{BP})\end{array}$ & $52 / 55$ & $0.114(0.194)$ & 1 \\
\hline & & $\begin{array}{l}\text { Arthroscopic } \\
\text { lavage versus } \\
\text { placebo }\end{array}$ & 121 & Pain & Unclear & $\begin{array}{c}\text { Knee } \\
\text { specific }\end{array}$ & & & \\
\hline & & & & & & Pain scale & $55 / 55$ & $0.110(0.236)$ & 2 \\
\hline & & & & & & $\begin{array}{l}\text { AIMS } \\
\text { (pain) }\end{array}$ & $56 / 55$ & $-0.200(0.223)$ & 1 \\
\hline & & & & & & $\begin{array}{l}\text { SF-36 } \\
\text { (BP) }\end{array}$ & $57 / 55$ & $0.090(0.189)$ & 3 \\
\hline
\end{tabular}


Table 1: Continued.

\begin{tabular}{|c|c|c|c|c|c|c|c|c|c|}
\hline Author & Journal & Comparison & $\begin{array}{c}\text { Number of } \\
\text { randomised } \\
\text { patients }\end{array}$ & Construct & $\begin{array}{l}\text { Selective } \\
\text { outcome } \\
\text { reporting }\end{array}$ & Outcome & $\begin{array}{l}\text { Number of } \\
\text { patients }\end{array}$ & $\begin{array}{c}\text { Standard mean } \\
\text { differences } \\
\text { (SMD) } \\
\text { measured as } \\
\text { Cohens'd (SE) }\end{array}$ & $\begin{array}{c}\text { Rank of } \\
\text { responsiveness } \\
\text { measured as } \\
\text { SMD }\end{array}$ \\
\hline \multirow[t]{4}{*}{$\begin{array}{l}\text { Neustadt } \\
\text { et al. } \\
2005\end{array}$} & J. Rheumatol. & $\begin{array}{l}\text { Hyalerone four } \\
\text { times a week } \\
\text { versus control }\end{array}$ & 251 & Pain & Unclear & WOMAC & $115 / 114$ & $0.098(0.162)$ & 2 \\
\hline & & & & & & $\begin{array}{c}\text { VAS } \\
\text { (activity) }\end{array}$ & $104 / 100$ & $0.158(0.172)$ & 1 \\
\hline & & $\begin{array}{l}\text { Hyalerone } \\
\text { three times a } \\
\text { week versus } \\
\text { control }\end{array}$ & 242 & Pain & Unclear & WOMAC & $107 / 114$ & $-0.028(0.164)$ & 2 \\
\hline & & & & & & $\begin{array}{c}\text { VAS } \\
\text { (activity) }\end{array}$ & $90 / 100$ & $0.030(0.176)$ & 1 \\
\hline \multirow[t]{2}{*}{$\begin{array}{l}\text { Niethard } \\
\text { et al. } \\
2005\end{array}$} & J. Rheumatol. & $\begin{array}{l}\text { Diclofena } \\
\text { versus placebo }\end{array}$ & 238 & Pain & Unclear & WOMAC & $117 / 120$ & $0.363(0.131)$ & 1 \\
\hline & & & & & & $\begin{array}{c}\text { VAS } \\
\text { (activity) }\end{array}$ & $117 / 119$ & $0.360(0.131)$ & 2 \\
\hline \multirow[t]{4}{*}{$\begin{array}{l}\text { Nuñez } \\
\text { et al. } \\
2006\end{array}$} & $\begin{array}{l}\text { Osteoarthritis } \\
\text { and Cartilage }\end{array}$ & $\begin{array}{l}\text { Education } \\
\text { programme } \\
\text { versus control }\end{array}$ & 100 & Pain & Unclear & WOMAC & $43 / 37$ & $0.980(0.237)$ & 1 \\
\hline & & & & & & $\begin{array}{l}\text { SF-36 } \\
\text { (BP) }\end{array}$ & $43 / 37$ & $0.605(0.229)$ & 2 \\
\hline & & & & Disability & & WOMAC & $43 / 37$ & $0.742(0.232)$ & 1 \\
\hline & & & & & & $\begin{array}{l}\text { SF-36 } \\
(\mathrm{PF})\end{array}$ & $43 / 37$ & $0.576(0.229)$ & 2 \\
\hline \multirow{2}{*}{$\begin{array}{l}\text { J. Petrella } \\
\text { and M. } \\
\text { Petrella } \\
2006\end{array}$} & $\begin{array}{l}\text { Arch. Intern. } \\
\text { Med. }\end{array}$ & $\begin{array}{l}\text { Massage versus } \\
\text { control }\end{array}$ & 74 & Pain & Adequate & WOMAC & $34 / 34$ & $0.948(0.256)$ & 1 \\
\hline & & & & & & $\begin{array}{l}\text { VAS } \\
\text { (knee) }\end{array}$ & $34 / 34$ & $0.872(0.254)$ & 2 \\
\hline \multirow[t]{5}{*}{$\begin{array}{l}\text { Petrella } \\
\text { et al. } \\
2006\end{array}$} & J. Rheumatol. & $\begin{array}{l}\text { Hyaleron } \\
\text { versuscontrol }\end{array}$ & 106 & Pain & Inadequate & WOMAC & $53 / 53$ & $0.092(0.194)$ & 1 \\
\hline & & & & & & $\begin{array}{c}\text { VAS } \\
\text { (activity) }\end{array}$ & $53 / 53$ & $-0.040(0.194)$ & 2 \\
\hline & & & & & & $\begin{array}{c}\text { VAS } \\
\text { (walking) }\end{array}$ & $53 / 53$ & $0.000(0.194)$ & 3 \\
\hline & & & & Disability & Unclear & WOMAC & $53 / 53$ & $-0.147(0.195)$ & 2 \\
\hline & & & & & & $\begin{array}{c}\text { SF-36 } \\
(\mathrm{PF})\end{array}$ & $53 / 53$ & $0.084(0.194)$ & 1 \\
\hline \multirow[t]{5}{*}{$\begin{array}{l}\text { Petrella } \\
2002\end{array}$} & $\begin{array}{l}\text { Arch. Intern. } \\
\text { Med. }\end{array}$ & $\begin{array}{l}\text { Hyalerone }+ \\
\text { placebo versus } \\
\text { control }\end{array}$ & 53 & Pain & Unclear & WOMAC & $25 / 28$ & $0.190(0.384)$ & 4 \\
\hline & & & & & & $\begin{array}{c}\text { VAS } \\
\text { (activity) }\end{array}$ & $25 / 28$ & $0.890(0.398)$ & 1 \\
\hline & & & & & & $\begin{array}{l}\text { VAS } \\
\text { (rest) }\end{array}$ & $25 / 28$ & $-0.539(0.389)$ & 3 \\
\hline & & & & & & $\begin{array}{c}\text { VAS } \\
\text { (walking) }\end{array}$ & $25 / 28$ & $0.623(0.391)$ & 2 \\
\hline & & $\begin{array}{l}\text { Hyalerone + } \\
\text { NSAID } \\
\text { versuscontrol }\end{array}$ & 57 & Pain & Unclear & WOMAC & $29 / 28$ & $0.238(0.377)$ & 4 \\
\hline
\end{tabular}


Table 1: Continued.

\begin{tabular}{|c|c|c|c|c|c|c|c|c|c|}
\hline Author & Journal & Comparison & $\begin{array}{c}\text { Number of } \\
\text { randomised } \\
\text { patients }\end{array}$ & Construct & $\begin{array}{l}\text { Selective } \\
\text { outcome } \\
\text { reporting }\end{array}$ & Outcome & $\begin{array}{l}\text { Number of } \\
\text { patients }\end{array}$ & $\begin{array}{l}\text { Standard mean } \\
\text { differences } \\
\text { (SMD) } \\
\text { measured as } \\
\text { Cohens'd (SE) }\end{array}$ & $\begin{array}{c}\text { Rank of } \\
\text { responsiveness } \\
\text { measured as } \\
\text { SMD }\end{array}$ \\
\hline & & & & & & $\begin{array}{c}\text { VAS } \\
\text { (activity) }\end{array}$ & $29 / 28$ & $0.501(0.381)$ & 2 \\
\hline & & & & & & $\begin{array}{l}\text { VAS } \\
\text { (rest) }\end{array}$ & $29 / 28$ & $0.383(0.379)$ & 3 \\
\hline & & $\begin{array}{l}\text { NSAID + saline } \\
\text { versus control }\end{array}$ & 54 & Pain & Unclear & $\begin{array}{c}\text { VAS } \\
\text { (walking) }\end{array}$ & $29 / 28$ & $1.268(0.403)$ & 1 \\
\hline & & & & & & WOMAC & $26 / 28$ & $0.336(0.384)$ & 3 \\
\hline & & & & & & $\begin{array}{c}\text { VAS } \\
\text { (activity) }\end{array}$ & $26 / 28$ & $1.622(0.428)$ & 1 \\
\hline & & & & & & $\begin{array}{l}\text { VAS } \\
\text { (rest) }\end{array}$ & $26 / 28$ & $0.173(0.382)$ & 4 \\
\hline & & & & & & $\begin{array}{c}\text { VAS } \\
\text { (walking) }\end{array}$ & $26 / 28$ & $1.154(0.406)$ & 2 \\
\hline \multirow[t]{4}{*}{$\begin{array}{l}\text { Pham } \\
\text { et al. } \\
2004\end{array}$} & $\begin{array}{l}\text { Ann. Rheum. } \\
\text { Dis. }\end{array}$ & $\begin{array}{l}\text { Hyalerone versus } \\
\text { control }\end{array}$ & 206 & Pain & Unclear & $\begin{array}{l}\text { VAS } \\
\text { (knee) }\end{array}$ & $131 / 85$ & $-0.035(0.177)$ & 2 \\
\hline & & & & & & $\begin{array}{l}\text { Painful } \\
\text { days }\end{array}$ & $131 / 85$ & $-0.078(0.177)$ & 1 \\
\hline & & $\begin{array}{l}\text { Diacerein versus } \\
\text { control }\end{array}$ & 170 & Pain & Unclear & $\begin{array}{c}\text { VAS } \\
\text { (knee) }\end{array}$ & $85 / 85$ & $-0.023(0.188)$ & 2 \\
\hline & & & & & & $\begin{array}{c}\text { Painful } \\
\text { days }\end{array}$ & $85 / 85$ & $-0.027(0.188)$ & 1 \\
\hline \multirow[t]{2}{*}{$\begin{array}{l}\text { Ravaud } \\
\text { et al. } \\
2009\end{array}$} & BMJ & $\begin{array}{l}\text { Standardised } \\
\text { consultation } \\
\text { versus usual care }\end{array}$ & 327 & Disability & Unclear & WOMAC & $146 / 181$ & $0.260(0.112)$ & 2 \\
\hline & & & & & & $\begin{array}{l}\text { SF-12 } \\
\text { (PCS) }\end{array}$ & $129 / 147$ & $0.361(0.122)$ & 1 \\
\hline \multirow[t]{2}{*}{$\begin{array}{l}\text { Raynauld } \\
\text { et al. } \\
2003\end{array}$} & $\begin{array}{l}\text { Arthritis } \\
\text { Rheum. }\end{array}$ & $\begin{array}{l}\text { Triamcinolone } \\
\text { versus saline } \\
\text { (control) }\end{array}$ & 68 & Pain & Unclear & WOMAC & $33 / 33$ & $0.307(0.248)$ & 2 \\
\hline & & & & & & $\begin{array}{l}\text { VAS } \\
\text { (knee) }\end{array}$ & $33 / 33$ & $0.672(0.253)$ & 1 \\
\hline \multirow[t]{2}{*}{$\begin{array}{l}\text { Raynauld } \\
\text { et al. } \\
2002\end{array}$} & $\begin{array}{l}\text { Osteoarthritis } \\
\text { and Cartilage }\end{array}$ & $\begin{array}{l}\text { Hylan G-F } 20 \\
\text { versus Control }\end{array}$ & 255 & Disability & Unclear & WOMAC & $124 / 107$ & $0.668(0.136)$ & 1 \\
\hline & & & & & & $\begin{array}{l}\text { SF-36 } \\
\text { (PCS) }\end{array}$ & $124 / 107$ & $0.553(0.134)$ & 2 \\
\hline \multirow[t]{3}{*}{$\begin{array}{l}\text { Rodrigues } \\
\text { et al. } \\
2008\end{array}$} & $\begin{array}{l}\text { Arthritis } \\
\text { Rheum. }\end{array}$ & $\begin{array}{l}\text { Medial-wedge } \\
\text { insole versus } \\
\text { neutral insole }\end{array}$ & 30 & Pain & Adequate & $\begin{array}{c}\text { VAS } \\
\text { (activity) }\end{array}$ & $16 / 14$ & $1.337(0.405)$ & 1 \\
\hline & & & & & & $\begin{array}{l}\text { VAS } \\
\text { (rest) }\end{array}$ & $16 / 14$ & $0.899(0.384)$ & 2 \\
\hline & & & & & & $\begin{array}{l}\text { VAS } \\
\text { (night) }\end{array}$ & $16 / 14$ & $0.802(0.380)$ & 3 \\
\hline \multirow[t]{4}{*}{$\begin{array}{l}\text { Rooks } \\
\text { et al. } \\
2006\end{array}$} & $\begin{array}{l}\text { Arthritis } \\
\text { Rheum }\end{array}$ & $\begin{array}{l}\text { Preoperative } \\
\text { exercise versus } \\
\text { control }\end{array}$ & 45 & Pain & Unclear & WOMAC & $14 / 15$ & $0.220(0.373)$ & 2 \\
\hline & & & & & & $\begin{array}{l}\text { SF-36 } \\
(\mathrm{BP})\end{array}$ & $14 / 15$ & $-0.322(0.374)$ & 1 \\
\hline & & & & Disability & & WOMAC & $14 / 15$ & $0.034(0.372)$ & 2 \\
\hline & & & & & & $\begin{array}{c}\text { SF-36 } \\
\text { (PF) }\end{array}$ & $14 / 15$ & $-0.391(0.375)$ & 1 \\
\hline
\end{tabular}


Table 1: Continued.

\begin{tabular}{|c|c|c|c|c|c|c|c|c|c|}
\hline Author & Journal & Comparison & $\begin{array}{c}\text { Number of } \\
\text { randomised } \\
\text { patients }\end{array}$ & Construct & $\begin{array}{l}\text { Selective } \\
\text { outcome } \\
\text { reporting }\end{array}$ & Outcome & $\begin{array}{c}\text { Number of } \\
\text { patients }\end{array}$ & $\begin{array}{l}\text { Standard mean } \\
\text { differences } \\
(\text { SMD) } \\
\text { measured as } \\
\text { Cohens'd (SE) }\end{array}$ & $\begin{array}{l}\text { Rank of } \\
\text { responsiveness } \\
\text { measured as } \\
\text { SMD }\end{array}$ \\
\hline \multirow[t]{4}{*}{$\begin{array}{l}\text { Sengupta } \\
\text { et al. } \\
2008\end{array}$} & $\begin{array}{l}\text { Arthritis Res. } \\
\text { Ther. }\end{array}$ & $\begin{array}{l}\text { 5-Loxin } \\
250 \mathrm{mg} \\
\text { versus } \\
\text { control }\end{array}$ & 50 & Pain & Unclear & WOMAC & $23 / 23$ & $1.292(0.393)$ & 2 \\
\hline & & & & & & $\begin{array}{c}\text { VAS } \\
\text { (knee) }\end{array}$ & $23 / 23$ & $2.463(0.467)$ & 1 \\
\hline & & $\begin{array}{l}\text { 5-Loxin } \\
100 \mathrm{mg} \\
\text { versus } \\
\text { control }\end{array}$ & 50 & Pain & Unclear & WOMAC & $24 / 23$ & $1.039(0.379)$ & 1 \\
\hline & & & & & & $\begin{array}{l}\text { VAS } \\
\text { (knee) }\end{array}$ & $24 / 23$ & $1.918(0.425)$ & 2 \\
\hline \multirow[t]{4}{*}{$\begin{array}{l}\text { Song } \\
\text { et al. } \\
2009\end{array}$} & $\begin{array}{l}\text { Ann. Rheum. } \\
\text { Dis. }\end{array}$ & $\begin{array}{l}\text { Icatibant } \\
2000 \mu \mathrm{g} \\
\text { versus } \\
\text { control }\end{array}$ & 29 & Pain & Unclear & $\begin{array}{c}\text { VAS } \\
\text { (activity) }\end{array}$ & $14 / 15$ & $0.492(0.459)$ & 1 \\
\hline & & & & & & $\begin{array}{l}\text { VAS } \\
\text { (rest) }\end{array}$ & $14 / 15$ & $0.391(0.456)$ & 2 \\
\hline & & $\begin{array}{l}\text { Icatibant } \\
500 \mu \mathrm{g} \\
\text { versus } \\
\text { control }\end{array}$ & 27 & Pain & Unclear & $\begin{array}{c}\text { VAS } \\
\text { (activity) }\end{array}$ & $12 / 15$ & $0.411(0.470)$ & 1 \\
\hline & & & & & & $\begin{array}{l}\text { VAS } \\
\text { (rest) }\end{array}$ & $12 / 15$ & $0.391(0.470)$ & 2 \\
\hline \multirow[t]{6}{*}{$\begin{array}{l}\text { Tannenbaum } \\
\text { et al. } \\
2004\end{array}$} & $\begin{array}{l}\text { Ann. Rheum. } \\
\text { Dis. }\end{array}$ & $\begin{array}{l}\text { Lumiracoxib } \\
400 \mathrm{mg} \\
\text { versus } \\
\text { placebo }\end{array}$ & 734 & Pain & Unclear & WOMAC & $491 / 243$ & $0.211(0.132)$ & 2 \\
\hline & & & & & & $\begin{array}{c}\text { VAS } \\
\text { (knee) }\end{array}$ & $491 / 243$ & $0.307(0.132)$ & 1 \\
\hline & & $\begin{array}{l}\text { Lumiracoxib } \\
200 \mathrm{mg} \\
\text { versus } \\
\text { placebo }\end{array}$ & 730 & Pain & Unclear & WOMAC & $487 / 243$ & $0.189(0.132)$ & 2 \\
\hline & & & & & & $\begin{array}{c}\text { VAS } \\
\text { (knee) }\end{array}$ & $487 / 243$ & $0.236(0.132)$ & 1 \\
\hline & & $\begin{array}{l}\text { Celecoxib } \\
\text { versus } \\
\text { placebo }\end{array}$ & 724 & Pain & Unclear & WOMAC & $481 / 243$ & $0.184(0.132)$ & 2 \\
\hline & & & & & & $\begin{array}{c}\text { VAS } \\
\text { (knee) }\end{array}$ & $481 / 243$ & $0.217(0.132)$ & 1 \\
\hline \multirow[t]{3}{*}{$\begin{array}{l}\text { Trnavský } \\
\text { et al. } \\
2004\end{array}$} & J. Rheumatol. & $\begin{array}{l}\text { Ibuprofen } \\
\text { versus } \\
\text { placebo }\end{array}$ & 50 & Pain & Unclear & $\begin{array}{c}\text { VAS } \\
\text { (walking) }\end{array}$ & $25 / 25$ & $1.739(0.332)$ & 1 \\
\hline & & & & & & $\begin{array}{l}\text { VAS } \\
\text { (rest) }\end{array}$ & $25 / 25$ & $0.932(0.298)$ & 2 \\
\hline & & & & & & $\begin{array}{c}\text { VAS } \\
\text { (knee) }\end{array}$ & $25 / 25$ & $0.786(0.294)$ & 3 \\
\hline \multirow[t]{2}{*}{$\begin{array}{l}\text { Vas } \\
\text { et al. } \\
2004\end{array}$} & BMJ & $\begin{array}{l}\text { Acupuncture } \\
\text { versus } \\
\text { control }\end{array}$ & 97 & Pain & Unclear & WOMAC & $48 / 49$ & $1.109(0.218)$ & 2 \\
\hline & & & & & & $\begin{array}{c}\text { VAS } \\
\text { (knee) }\end{array}$ & $48 / 49$ & $1.249(0.222)$ & 1 \\
\hline
\end{tabular}


TABle 1: Continued.

\begin{tabular}{|c|c|c|c|c|c|c|c|c|c|}
\hline Author & Journal & Comparison & $\begin{array}{c}\text { Number of } \\
\text { randomised } \\
\text { patients }\end{array}$ & Construct & $\begin{array}{l}\text { Selective } \\
\text { outcome } \\
\text { reporting }\end{array}$ & Outcome & $\begin{array}{l}\text { Number of } \\
\text { patients }\end{array}$ & $\begin{array}{l}\text { Standard mean } \\
\text { differences } \\
(\text { SMD) } \\
\text { measured as } \\
\text { Cohens'd (SE) }\end{array}$ & $\begin{array}{l}\text { Rank of } \\
\text { responsiveness } \\
\text { measured as } \\
\text { SMD }\end{array}$ \\
\hline \multirow[t]{4}{*}{$\begin{array}{l}\text { Wang } \\
\text { et al. } \\
2009\end{array}$} & $\begin{array}{l}\text { Arthritis } \\
\text { Rheum. }\end{array}$ & $\begin{array}{l}\text { Tai chi versus } \\
\text { control }\end{array}$ & 40 & Pain & Adequate & WOMAC & $20 / 20$ & $0.456(0.320)$ & 1 \\
\hline & & & & & & $\begin{array}{c}\text { VAS } \\
\text { (knee) }\end{array}$ & $20 / 20$ & $0.015(0.316)$ & 2 \\
\hline & & & 40 & Disability & Adequate & WOMAC & $20 / 20$ & $0.356(0.319)$ & 2 \\
\hline & & & & & & $\begin{array}{l}\text { SF-36 } \\
\text { (PCS) }\end{array}$ & $20 / 20$ & $0.820(0.329)$ & 1 \\
\hline \multirow[t]{5}{*}{$\begin{array}{l}\text { Witt } \\
\text { et al. } \\
2005\end{array}$} & Lancet & $\begin{array}{l}\text { Acupuncture } \\
\text { versus } \\
\text { minimal } \\
\text { acupuncture }\end{array}$ & 226 & Pain & Unclear & WOMAC & $149 / 75$ & $0.150(0.142)$ & 1 \\
\hline & & & & & & SES & $149 / 75$ & $0.062(0.142)$ & 2 \\
\hline & & & 226 & Disability & Unclear & WOMAC & $149 / 75$ & $0.249(0.142)$ & 1 \\
\hline & & & & & & $\begin{array}{l}\text { SF-36 } \\
\text { (PCS) }\end{array}$ & $149 / 75$ & $0.215(0.142)$ & 3 \\
\hline & & & & & & PDI & $149 / 75$ & $0.245(0.142)$ & 2 \\
\hline \multirow[t]{4}{*}{$\begin{array}{l}\text { Wittenberg } \\
\text { et al. } \\
2006\end{array}$} & $\begin{array}{l}\text { Arthritis } \\
\text { Rheum. }\end{array}$ & $\begin{array}{l}\text { Lumiracoxib } \\
\text { versus } \\
\text { placebo }\end{array}$ & 219 & Pain & Unclear & WOMAC & $144 / 75$ & $0.406(0.185)$ & 2 \\
\hline & & & & & & $\begin{array}{c}\text { VAS } \\
\text { (activity) }\end{array}$ & $144 / 75$ & $0.645(0.186)$ & 1 \\
\hline & & $\begin{array}{l}\text { Celecoxib } \\
\text { versus } \\
\text { placebo }\end{array}$ & 220 & Pain & Unclear & WOMAC & $145 / 75$ & $0.396(0.184)$ & 2 \\
\hline & & & & & & $\begin{array}{c}\text { VAS } \\
\text { (activity) }\end{array}$ & $145 / 75$ & $0.525(0.185)$ & 1 \\
\hline
\end{tabular}

WOMAC (Western Ontario and McMaster Universities Arthritis Index), VAS (visual analogue scale), NRS (numerical rating scale), AIMS (Arthritis Impact Measurement Scale), ASES (Arthritis Self-Efficacy Scale), HAQ (Health Assessment Questionnaire), PDI (Pain Disability Index), SES (Schmerzempfindungsskala) (Pain Experience Scale), SF-36 (Short Form 36), BP (bodily pain), PF (physical function), PCS (Physical Composite Scale).

\section{Discussion}

4.1. Summary of Evidence. Biased selection of PROs in metaanalyses (e.g., choosing the most favourable PROs for the intervention from individual trials) can overestimate the effect compared with a systematic approach. As anticipated, choosing the "most favourable" outcome from each individual trial was more positive than systematic approaches based on either (1) how frequently the PROs were used, (2) the average responsiveness of the PROs, or (3) the developed list. Using a prioritised list as developed in this study is recommended to reduce the risk of biased selection of PROs in meta-analyses. When comparing the list developed in this study with the hierarchy for extracting pain measurement scales published by Jüni et al. [11], the main differences are that Jüni et al. included global health outcome (as the total WOMAC score, patient's global health and physician's global health), contrasting with the developed list in this study, which only included specific pain measurement scales. The list used by Bartels et al. [12] was based on consensus among the authors and reports the WOMAC subscales as first choice in both pain and disability, similar to the list developed in this study.

4.2. Limitations. This study has some limitations. First of all, even though 38 trials were included, and 35 trials had sufficient data on pain, only 15 trials had sufficient data on disability. The literature search was performed in the ten highest impact factor general and internal medicine journals as well as the ten highest impact factor rheumatology journals in order to identify trials with a low risk of selective reporting bias. Some of the highly ranked journals were primarily review journals. Even though these journals only published a few randomised controlled trials, some of the eligible trials were published in one of these journals. Only six trials were classified as "adequate" for having a low risk of selective reporting bias, and some frequently used PROs were missing in the list based on these trials. However, as the differences between the list based on these trials with a low risk of selective outcome reporting bias and the list based on all the included 38 trials were very small, the developed 
TABLE 2: Frequency of the used outcomes listed according to their frequency in the included trials.

\begin{tabular}{|c|c|c|c|}
\hline Pain outcome (scale) & $\begin{array}{c}\text { Number of trials } \\
k=35(\%)\end{array}$ & $\begin{array}{c}\text { Number of substudies } \\
k=54(\%)\end{array}$ & Mean rank in responsiveness (range) \\
\hline WOMAC pain (Likert scale) & $15(43 \%)$ & $20(37 \%)$ & $1.8(1-4)$ \\
\hline Global knee pain (VAS) & $13(37 \%)$ & $17(31 \%)$ & $1.7(1-4)$ \\
\hline WOMAC pain (100 mm scale) & $12(34 \%)$ & $21(39 \%)$ & $1.9(1-4)$ \\
\hline Pain during activity (VAS) & $12(34 \%)$ & $18(33 \%)$ & $1.4(1-5)$ \\
\hline Pain during walking (VAS) & $7(20 \%)$ & $12(22 \%)$ & $1.5(1-3)$ \\
\hline Pain at rest (VAS) & $7(20 \%)$ & $12(22 \%)$ & $2.3(1-4)$ \\
\hline SF-36 (bodily pain (BP) subscale) & $6(17 \%)$ & $8(15 \%)$ & $2.3(1-4)$ \\
\hline Pain at night (VAS) & $2(6 \%)$ & $4(7 \%)$ & $3.0(3)$ \\
\hline HAQ (pain subscale) & $1(3 \%)$ & $4(7 \%)$ & $1.5(1-2)$ \\
\hline $\begin{array}{l}\text { Lequesne algofunctional index } \\
\text { (pain subscale) }\end{array}$ & $1(3 \%)$ & $2(4 \%)$ & $1.5(1-2)$ \\
\hline Pain during activity (NRS) & $1(3 \%)$ & $2(4 \%)$ & $2.5(1-4)$ \\
\hline Pain during walking (NRS) & $1(3 \%)$ & $2(4 \%)$ & $2.5(2-3)$ \\
\hline Number of painful days (days) & $1(3 \%)$ & $2(4 \%)$ & $1.0(1)$ \\
\hline AIMS (pain subscale) & $1(3 \%)$ & $2(4 \%)$ & $1.5(1-2)$ \\
\hline Knee-Specific Pain Scale (KSPS) & $1(3 \%)$ & $2(4 \%)$ & $2.5(2-3)$ \\
\hline $\begin{array}{l}\text { McGill Pain Questionnaire (pain } \\
\text { intensity) }\end{array}$ & $1(3 \%)$ & $1(2 \%)$ & $2.0(2)$ \\
\hline ASES (pain subscale) & $1(3 \%)$ & $1(2 \%)$ & $2.0(2)$ \\
\hline SES (Schmerzempfindungsskala) & $1(3 \%)$ & $1(2 \%)$ & $2.0(2)$ \\
\hline Disability outcome (scale) & $\begin{array}{c}\text { Number of trials } \\
k=15(\%)\end{array}$ & $\begin{array}{c}\text { Number of substudies } \\
k=21(\%)\end{array}$ & Mean rank in responsiveness (range) \\
\hline $\begin{array}{l}\text { Physical composite score (PFC) } \\
\text { (based on SF-36, SF-12, or SF-8) }\end{array}$ & $9(60 \%)$ & $11(52 \%)$ & $1.8(1-3)$ \\
\hline WOMAC function (100 mm scale) & $8(53 \%)$ & $13(62 \%)$ & $1.5(1-2)$ \\
\hline WOMAC function (Likert scale) & $7(47 \%)$ & $8(38 \%)$ & $1.5(1-2)$ \\
\hline $\begin{array}{l}\text { SF-36 (physical function PF } \\
\text { subscale) }\end{array}$ & $7(47 \%)$ & $8(38 \%)$ & $1.8(1-2)$ \\
\hline HAQ (disability subscale) & $1(7 \%)$ & $4(19 \%)$ & $1.5(1-2)$ \\
\hline PDI (pain disability index) & $1(7 \%)$ & $1(5 \%)$ & $2.0(2)$ \\
\hline ASES (disability subscale) & $1(7 \%)$ & $1(5 \%)$ & $2.0(2)$ \\
\hline
\end{tabular}

WOMAC (Western Ontario and McMaster Universities Arthritis Index), VAS (visual analogue scale), NRS (Numerical Rating Scale), AIMS (Arthritis Impact Measurement Scale), ASES (Arthritis Self Efficacy Scale), HAQ (Health Assessment Questionnaire), PDI (Pain Disability Index), SES (Schmerzempfindungsskala) (Pain Experience Scale), SF-36 (Short Form 36), SF-12 (Short Form 12), SF-8 (Short Form 8).

list seems to be trustworthy. Furthermore, stratified analyses comparing lists based on the subgroups of trials and the list from all included trials showed only small discrepancies for the subgroup of "oral medication" using pain as outcome. No differences were seen in other subgroup analyses.

As the subgroup analyses based on whether the trials were published in the general/internal medicine journals or in the rheumatology journals showed no differences between the list from the subgroup and the list from all included trials, it seems unlikely that including more trials will change the list of the most frequently used outcome. So, even though including more journals in the literature search could be preferable, based on these subgroup analyses, it is unlikely that including more trials would change the list developed in this study.
Secondly, combining the SMDs across different populations and interventions can cause heterogeneity, as characteristics of the patient populations (age, sex, BMI, etc.) and interventions have an impact on the effect size. A large SMD can firstly be due to a large difference in mean change between the intervention group and control group, or secondly, to a small standard deviation due to small variability in the included patient group. Combining SMDs across trials could then cause bias if trials with a homogeneous patient group (small SD) were combined with trials with a heterogeneous patient group (large SD). These differences are reduced when using the rank of the SMD instead, when comparing across trials. In developing the prioritised list for extracting PROs in this study, the estimated SMDs were then only used for ranking the PROs 


\section{Different approaches to extracting pain outcome}

The recommended list

Scenario analysis number 1 most favourable outcome in individual trials

Scenario analysis number 2 most responsive outcome on average

Scenario analysis number 3 most frequently used outcome

$\begin{array}{ccc}\begin{array}{c}\text { Pooled } \\ \text { SMD }\end{array} & \begin{array}{c}\text { Lower } \\ \text { limit }\end{array} & \begin{array}{c}\text { Upper } \\ \text { limit }\end{array} \\ 0.298 & 0.224 & 0.372 \\ 0.435 & 0.339 & 0.531 \\ 0.388 & 0.296 & 0.480 \\ 0.298 & 0.224 & 0.372 \\ & & \\ & & \\ \text { Pooled } & \text { Lower } & \text { Upper } \\ \text { SMD } & \text { limit } & \text { limit } \\ 0.323 & 0.237 & 0.409 \\ 0.416 & 0.330 & 0.502 \\ 0.381 & 0.297 & 0.465 \\ 0.316 & 0.230 & 0.402\end{array}$

SMD 95\% CI
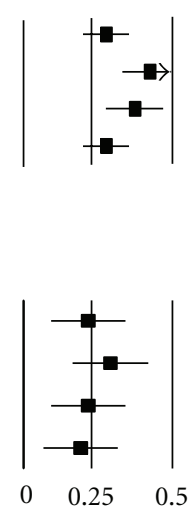

FiguRE 2: Impact on different approaches to extracting data for meta-analysis.

TABle 3: A prioritized list of patient-reported outcomes for extracting data in meta-analysis.

\section{Pain}

outcome (scale)

(1) WOMAC pain subscale (Likert/100 mm)

(2) Pain during activity (VAS)

(3) Pain during walking (VAS)

(4) Global knee pain (VAS)

(5) Pain at rest (VAS)

(6) SF-36 (bodily pain (BP) subscale)

(7) HAQ (pain subscale), Lequesne algofunctional index (pain subscale), AIMS (pain subscale), Knee-Specific Pain Scale (KSPS), McGill Pain Questionnaire (pain intensity), ASES (pain subscale), SES (Schmerzempfindungsskala)

(8) Pain at night (VAS), pain during activity (NRS), pain on walking (NRS), number of painful days (days)

Disability

outcome (scale)

(1) WOMAC subscale function (Likert/100 mm)

(2) SF-36 (subscale physical function (PF))

(3) Physical composite score (PCS) based on SF-36, SF-12, or SF-8

(4) HAQ (disability subscale), PDI (pain disability index), ASES (disability subscale)

WOMAC (Western Ontario and McMaster Universities Arthritis Index), VAS (visual analogue scale), NRS (Numerical Rating Scale), AIMS (Arthritis impact measurement scale), ASES (Arthritis Self Efficacy Scale), HAQ (health assessment questionnaire), PDI (Pain Disability Index), SES (Schmerzempfindungsskala) (Pain Experience Scale), SF-36 (Short Form 36), SF-12 (Short Form 12), SF-8 (Short Form 8).

in each trial. When the variability in a trial was small due to a homogeneous patient group, the variability in all PROs in the trial was then expected to be equally small with the rank of the SMD still being an acceptable measure of the relationship of the responsiveness between the PROs in individual trials.

Thirdly, as a large number of different PROs were used and most of the included trials only compared two PROs on either pain or disability, it was not possible to make direct comparisons of the responsiveness of PROs. Therefore, the indirect method of ranking the PROs in the individual trials according to responsiveness was used. This method of ranking the PROs reduces the impact of the differences in populations and interventions between the included trials, as it only used the SMD for ranking the responsiveness of the PROs. As the mean rank of the PROs could change if more trials were included, especially the PROs only used in a few trials, the systematically developed list in this study was based on the most frequently used PROs (used in at least 5 trials).

4.3. Strengths. A strength of this study was that a comprehensive systematic literature search in high-quality journals was performed, and a systematic approach was used for developing the prioritised list. Furthermore, the impact of using the developed list for data extraction in meta-analyses was analysed. Finally, the prioritised list developed in this study was compared with a list based on the six trials with a low risk of selective outcome reporting bias, and the differences were small.

To summarise, the WOMAC subscales for both pain and disability should be the first choice for extracting PROs in meta-analyses for patients with knee OA. Different single item VAS scores were actually measuring different items (e.g., VAS during activity covers "pain during daily activity," "pain following stepping activities," or "pain during worst activity"), so, even though different single item VAS scores for measuring pain were more responsive than the WOMAC, WOMAC is preferred for meta-analysis.

The Knee Injury and Osteoarthritis Outcome Score (KOOS) "ADL" subscale is equivalent to the WOMAC "function" subscale, and the WOMAC "pain" score is contained in the KOOS "pain" score. As the corresponding KOOS subscales and WOMAC subscales have shown equal responsiveness [57], the KOOS subscales could be included in the developed list. Based on these preliminary results and considerations, we recommend the list presented in Table 3, when extracting PROs on pain and disability for meta-analysis. 


\section{Conclusions}

As choosing the most favorable patient-reported outcomes (PROs) from individual trials can overestimate the effect compared with a systematic approach, using a prioritised list as presented in this study is recommended to reduce reviewers' likelihood of biased selection of PROs in meta-analyses.

The impact of the prioritised list should be tested in published meta-analyses investigating the effect of interventions on pain and disability for patients with knee osteoarthritis. When a larger number of trials are registered and classified as "adequate" for having a low risk of selective reporting outcome bias, this study should be repeated in order to check the prioritised list based on the responsiveness of PROs.

\section{Acknowledgments}

The authors thank The Health Insurance Foundation, Danish Physiotherapy Association, University of Southern Denmark's Research Unit for Musculoskeletal Function and Physiotherapy, and Department of Physiotherapy, University Hospital, Gentofte. The Musculoskeletal Statistics Unit at The Parker Institute is supported by grants from the Oak Foundation.

\section{References}

[1] M. Dougados, "Monitoring osteoarthritis progression and therapy," Osteoarthritis and Cartilage, vol. 12, pp. S55-S60, 2004.

[2] T. E. McAlindon, M. P. La Valley, J. P. Gulin, and D. T. Felson, "Glucosamine and chondroitin for treatment of osteoarthritis: a systematic quality assessment and meta-analysis," Journal of the American Medical Association, vol. 283, no. 11, pp. 1469$1475,2000$.

[3] M. Fransen and S. McConnell, "Exercise for osteoarthritis of the knee," Cochrane Database of Systematic Reviews, no. 4, Article ID CD004376, 2008.

[4] J. L. Hutton and P. R. Williamson, "Bias in meta-analysis due to outcome variable selection within studies," Journal of the Royal Statistical Society C, vol. 49, no. 3, pp. 359-370, 2000.

[5] J. J. Kirkham, D. G. Altman, and P. R. Williamson, "Bias due to changes in specified outcomes during the systematic review process," PloS ONE, vol. 5, no. 3, Article ID e9810, 2010.

[6] L. B. Mokkink, C. B. Terwee, D. L. Patrick et al., "The COSMIN study reached international consensus on taxonomy, terminology, and definitions of measurement properties for health-related patient-reported outcomes," Journal of Clinical Epidemiology, vol. 63, no. 7, pp. 737-745, 2010.

[7] M. A. Puhan, I. Soesilo, G. H. Guyatt, and H. J. Schünemann, "Combining scores from different patient reported outcome measures in meta-analyses: when is it justified?" Health and Quality of Life Outcomes, vol. 4, article 94, 2006.

[8] C. O. Bingham III, C. Pohl, R. Alten et al., "Flare and disease worsening in rheumatoid arthritis: time for a definition," International Journal of Advances in Rheumatology, vol. 7, no. 3, pp. 85-91, 2007.

[9] N. Bellamy, J. Kirwan, M. Boers et al., "Recommendations for a core set of outcome measures for future phase III clinical trials in knee, hip, and hand osteoarthritis. Consensus development at OMERACT III," Journal of Rheumatology, vol. 24, no. 4, pp. 799-802, 1997.

[10] B. C. Johnston, K. Thorlund, H. J. Schünemann et al., "Improving the interpretation of quality of life evidence in metaanalyses: the application of minimal important difference units," Health and Quality of Life Outcomes, vol. 8, article 116, 2010.

[11] P. Jüni, S. Reichenbach, and P. Dieppe, "Osteoarthritis: rational approach to treating the individual," Best Practice and Research, vol. 20, no. 4, pp. 720-740, 2006.

[12] E. M. Bartels, H. Lund, K. B. Hagen, H. Dagfinrud, R. Christensen, and B. Danneskiold-Samsøe, "Aquatic exercise for the treatment of knee and hip osteoarthritis," Cochrane Database of Systematic Reviews, no. 4, Article ID CD005523, 2007.

[13] R. Altman, E. Asch, and D. Bloch, "Development of criteria for the classification and reporting of osteoarthritis. Classification of osteoarthritis of the knee," Arthritis and Rheumatism, vol. 29, no. 8, pp. 1039-1052, 1986.

[14] L. Lepage, D. G. Altman, K. F. Schulz et al., "The revised CONSORT statement for reporting randomized trials: explanation and elaboration," Annals of Internal Medicine, vol. 134, no. 8, pp. 663-694, 2001.

[15] K. P. Lee, M. Schotland, P. Bacchetti, and L. A. Bero, "Association of journal quality indicators with methodological quality of clinical research articles," Journal of the American Medical Association, vol. 287, no. 21, pp. 2805-2808, 2002.

[16] J. P. T. Higgins and S. Green, Eds., Cochrane Handbook for Systematic Reviews of Interventions, Version 5.1.0, The Cochrane Collaboration, 2011.

[17] J. P. T. Higgins, S. G. Thompson, J. J. Deeks, and D. G. Altman, "Measuring inconsistency in meta-analyses," British Medical Journal, vol. 327, no. 7414, pp. 557-560, 2003.

[18] R. D. Altman, J. E. Rosen, D. A. Bloch, H. T. Hatoum, and P. Korner, "A double-blind, randomized, saline-controlled study of the efficacy and safety of EUFLEXXA® for treatment of painful osteoarthritis of the knee, with an open-label safety extension (The FLEXX Trial)," Seminars in Arthritis and Rheumatism, vol. 39, no. 1, pp. 1-9, 2009.

[19] A. W. A. Baltzer, C. Moser, S. A. Jansen, and R. Krauspe, "Autologous conditioned serum (Orthokine) is an effective treatment for knee osteoarthritis," Osteoarthritis and Cartilage, vol. 17, no. 2, pp. 152-160, 2009.

[20] H. R. Barthel, D. Haselwood, S. Longley III, M. S. Gold, and R. D. Altman, "Randomized controlled trial of diclofenac sodium gel in knee osteoarthritis," Seminars in Arthritis and Rheumatism, vol. 39, no. 3, pp. 203-212, 2009.

[21] K. L. Bennell, R. S. Hinman, B. R. Metcalf et al., "Efficacy of physiotherapy management of knee joint osteoarthritis: a randomised, double blind, placebo controlled trial," Annals of the Rheumatic Diseases, vol. 64, no. 6, pp. 906-912, 2005.

[22] B. M. Berman, L. Lao, P. Langenberg, W. L. Lee, A. M. K. Gilpin, and M. C. Hochberg, "Effectiveness of acupuncture as adjunctive therapy in osteoarthritis of the knee: a randomized, controlled trial," Annals of Internal Medicine, vol. 141, no. 12, pp. 901-910, 2004.

[23] F. X. Burch, J. N. Tarro, J. J. Greenberg, and W. J. Carroll, "Evaluating the benefits of patterned stimulation in the treatment of osteoarthritis of the knee. A multi-center, randomized, single-blind, controlled study with an independent masked evaluator," Osteoarthritis and Cartilage, vol. 16, no. 8, pp. 865872, 2008. 
[24] J. P. Case, A. J. Baliunas, and J. A. Block, "Lack of efficacy of acetaminophen in treating symptomatic knee osteoarthritis: a randomized, double-blind, placebo-controlled comparison trial with diclofenac sodium," Archives of Internal Medicine, vol. 163, no. 2, pp. 169-178, 2003.

[25] D. O. Clegg, D. J. Reda, C. L. Harris et al., "Glucosamine, chondroitin sulfate, and the two in combination for painful knee osteoarthritis," The New England Journal of Medicine, vol. 354, no. 8, pp. 795-808, 2006.

[26] R. Forestier, H. Desfour, J. M. Tessier et al., "Spa therapy in the treatment of knee osteoarthritis: a large randomised multicentre trial," Annals of the Rheumatic Diseases, vol. 69, no. 4, pp. 660-665, 2010.

[27] G. P. Geba, A. L. Weaver, A. B. Polis, M. E. Dixon, and T. J. Schnitzer, "Efficacy of rofecoxib, celecoxib, and acetaminophen in osteoarthritis of the knee: a randomized trial," Journal of the American Medical Association, vol. 287, no. 1, pp. 64-71, 2002.

[28] A. Gibofsky, G. W. Williams, F. McKenna, and J. G. Fort, "Comparing the efficacy of cyclooxygenase 2-specific inhibitors in treating osteoarthritis: appropriate trial design considerations and results of a randomized, placebo-controlled trial," Arthritis and Rheumatism, vol. 48, no. 11, pp. 31023111, 2003.

[29] R. S. Hinman, K. M. Crossley, J. McConnell, and K. L. Bennell, "Efficacy of knee tape in the management of osteoarthritis of the knee: blinded randomised controlled trial," British Medical Journal, vol. 327, no. 7407, pp. 135-138, 2003.

[30] R. Hughes and A. Carr, "A randomized, double-blind, placebo-controlled trial of glucosamine sulphate as an analgesic in osteoarthritis of the knee," Rheumatology, vol. 41, no. 3, pp. 279-284, 2002.

[31] A. Kirkley, T. B. Birmingham, R. B. Litchfield et al., "A randomized trial of arthroscopic surgery for osteoarthritis of the knee," The New England Journal of Medicine, vol. 359, no. 11, pp. 1097-1107, 2008.

[32] G. S. Kitay, M. J. Koren, D. L. Helfet, M. K. Parides, and J. A. Markenson, "Efficacy of combined local mechanical vibrations, continuous passive motion and thermotherapy in the management of osteoarthritis of the knee," Osteoarthritis and Cartilage, vol. 17, no. 10, pp. 1269-1274, 2009.

[33] B. Mazières, M. Hucher, M. Zaïm, and P. Garnero, "Effect of chondroitin sulphate in symptomatic knee osteoarthritis: a multicentre, randomised, double-blind, placebo-controlled study," Annals of the Rheumatic Diseases, vol. 66, no. 5, pp. 639-645, 2007.

[34] C. J. McCarthy, P. M. Mills, R. Pullen, C. Roberts, A. Silman, and J. A. Oldham, "Supplementing a home exercise programme with a class-based exercise programme is more effective than home exercise alone in the treatment of knee osteoarthritis," Rheumatology, vol. 43, no. 7, pp. 880-886, 2004.

[35] J. B. Moseley, K. O’Malley, N. J. Petersen et al., “A controlled trial of arthroscopic surgery for osteoarthritis of the knee," The New England Journal of Medicine, vol. 347, no. 2, pp. 81-88, 2002.

[36] D. Neustadt, J. Caldwell, M. Bell, J. Wade, and J. Gimbel, "Clinical effects of intraarticular injection of high molecular

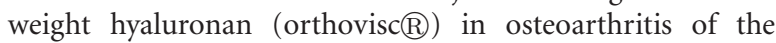
knee: a randomized, controlled, multicenter trial," Journal of Rheumatology, vol. 32, no. 10, pp. 1928-1936, 2005.

[37] F. U. Niethard, M. S. Gold, G. S. Solomon et al., "Efficacy of topical diclofenac diethylamine gel in osteoarthritis of the knee," Journal of Rheumatology, vol. 32, no. 12, pp. 2384-2392, 2005.

[38] M. Nuñez, E. Nuñez, J. M. Segur et al., "The effect of an educational program to improve health-related quality of life in patients with osteoarthritis on waiting list for total knee replacement: a randomized study," Osteoarthritis and Cartilage, vol. 14, no. 3, pp. 279-285, 2006.

[39] A. I. Perlman, A. Sabina, A. L. Williams, V. Y. Njike, and D. L. Katz, "Massage therapy for osteoarthritis of the knee: a randomized controlled trial," Archives of Internal Medicine, vol. 166, no. 22, pp. 2533-2538, 2006.

[40] R. J. Petrella and M. Petrella, "A prospective, randomized, double-blind, placebo controlled study to evaluate the efficacy of intraarticular hyaluronic acid for osteoarthritis of the knee," Journal of Rheumatology, vol. 33, no. 5, pp. 951-956, 2006.

[41] R. J. Petrella, M. D. DiSilvestro, and C. Hildebrand, "Effects of hyaluronate sodium on pain and physical functioning in osteoarthritis of the knee: a randomized, double-blind, placebo-controlled clinical trial," Archives of Internal Medicine, vol. 162, no. 3, pp. 292-298, 2002.

[42] T. Pham, A. Le Henanff, P. Ravoud, P. Dieppe, L. Paolozzi, and M. Dougados, "Evaluation of the symptomatic and structural efficacy of a new hyaluronic acid compound, NRD101, in comparison with diacerein and placebo in a 1 year randomised controlled study in symptomatic knee osteoarthritis," Annals of the Rheumatic Diseases, vol. 63, no. 12, pp. 1611-1617, 2004.

[43] P. Ravaud, R. M. Flipo, I. Boutron et al., "ARTIST (osteoarthritis intervention standardized) study of standardised consultation versus usual care for patients with osteoarthritis of the knee in primary care in France: pragmatic randomised controlled trial," British Medical Journal, vol. 338, no. 7696, article b421, 2009.

[44] J. P. Raynauld, C. H. Goldsmith, N. Bellamy et al., "Effectiveness and safety of repeat courses of hylan G-F 20 in patients with knee osteoarthritis," Osteoarthritis and Cartilage, vol. 13, no. 2, pp. 111-119, 2005.

[45] J. P. Raynauld, C. Buckland-Wright, R. Ward et al., "Safety and efficacy of long-term intraarticular steroid injections in osteoarthritis of the knee: a randomized, double-blind, placebo-controlled trial," Arthritis and Rheumatism, vol. 48, no. 2, pp. 370-377, 2003.

[46] P. T. Rodrigues, A. F. Ferreira, R. M. R. Pereira, E. Bonfá, E. F. Borba, and R. Fuller, "Effectiveness of medial-wedge insole treatment for valgus knee osteoarthritis," Arthritis Care and Research, vol. 59, no. 5, pp. 603-608, 2008.

[47] D. S. Rooks, J. Huang, B. E. Bierbaum et al., "Effect of preoperative exercise on measures of functional status in men and women undergoing total hip and knee arthroplasty," Arthritis Care and Research, vol. 55, no. 5, pp. 700-708, 2006.

[48] K. Sengupta, K. V. Alluri, A. R. Satish et al., "A double blind, randomized, placebo controlled study of the efficacy and safety of 5-Loxin $\AA$ for treatment of osteoarthritis of the knee," Arthritis Research and Therapy, vol. 10, no. 4, article R85, 2008.

[49] I. H. Song, C. E. Althoff, K. G. Hermann et al., "Contrastenhanced ultrasound in monitoring the efficacy of a bradykinin receptor 2 antagonist in painful knee osteoarthritis compared with MRI," Annals of the Rheumatic Diseases, vol. 68 , no. 1 , pp. 75-83, 2009.

[50] H. Tannenbaum, F. Berenbaum, J. Y. Reginster et al., "Lumiracoxib is effective in the treatment of osteoarthritis of the knee: a 13 week, randomised, double blind study versus placebo and celecoxib," Annals of the Rheumatic Diseases, vol. 63, no. 11, pp. 1419-1426, 2004. 
[51] K. Trnavský, M. Fischer, U. Vögtle-Junkert, and F. Schreyger, "Efficacy and safety of 5\% ibuprofen cream treatment in knee osteoarthritis. results of a randomized, double-blind, placebocontrolled study," Journal of Rheumatology, vol. 31, no. 3, pp. 565-572, 2004.

[52] J. Vas, C. Méndez, E. Perea-Milla et al., "Acupuncture as a complementary therapy to the pharmacological treatment of osteoarthritis of the knee: randomised controlled trial," British Medical Journal, vol. 329, no. 7476, pp. 1216-1219, 2004.

[53] C. Wang, C. H. Schmid, P. L. Hibberd et al., "Tai Chi is effective in treating knee osteoarthritis: a randomized controlled trial," Arthritis Care and Research, vol. 61, no. 11, pp. 1545-1553, 2009.

[54] C. Witt, B. Brinkhaus, S. Jena et al., "Acupuncture in patients with osteoarthritis of the knee: a randomised trial," The Lancet, vol. 366, no. 9480, pp. 136-143, 2005.

[55] R. H. Wittenberg, E. Schell, G. Krehan et al., "First-dose analgesic effect of the cyclo-oxygenase-2 selective inhibitor lumiracoxib in osteoarthritis of the knee: a randomized, double-blind, placebo-controlled comparison with celecoxib [NCT00267215]," Arthritis Research and Therapy, vol. 8, no. 2, article R35, 2006.

[56] US Department of Health and Human Services Food and Drug Administration (FDA), Guidance for Industry: PatientReported Outcome Measures: Use in Medical Product Development to Support Labelling Claims, Food and Drug Administration, 2009.

[57] A. M. Davis, L. S. Lohmander, R. Wong, V. Venkataramanan, and G. A. Hawker, "Evaluating the responsiveness of the ICOAP following hip or knee replacement," Osteoarthritis and Cartilage, vol. 18, no. 8, pp. 1043-1045, 2010. 


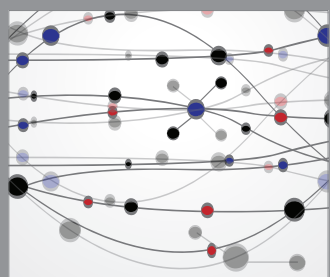

The Scientific World Journal
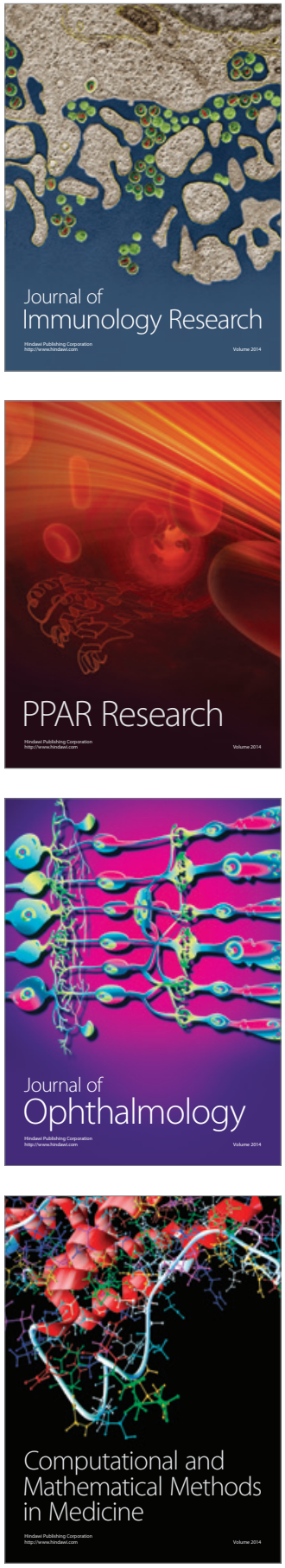

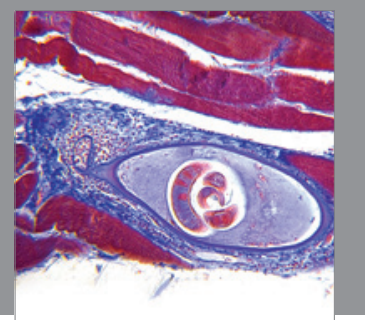

Gastroenterology

Research and Practice
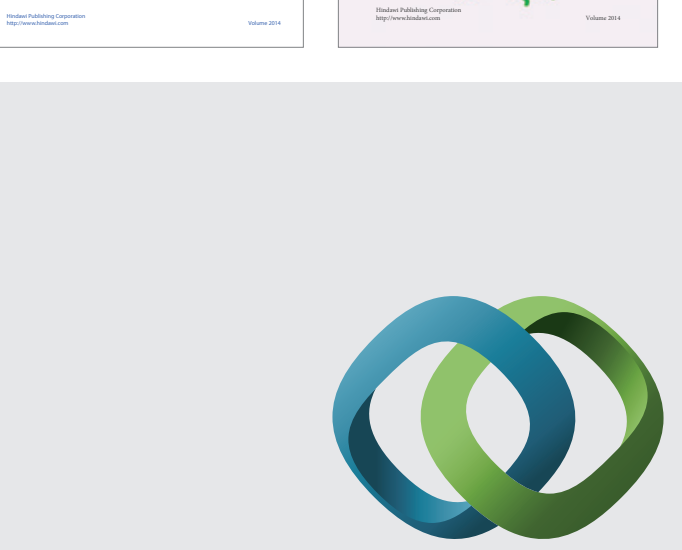

\section{Hindawi}

Submit your manuscripts at

http://www.hindawi.com
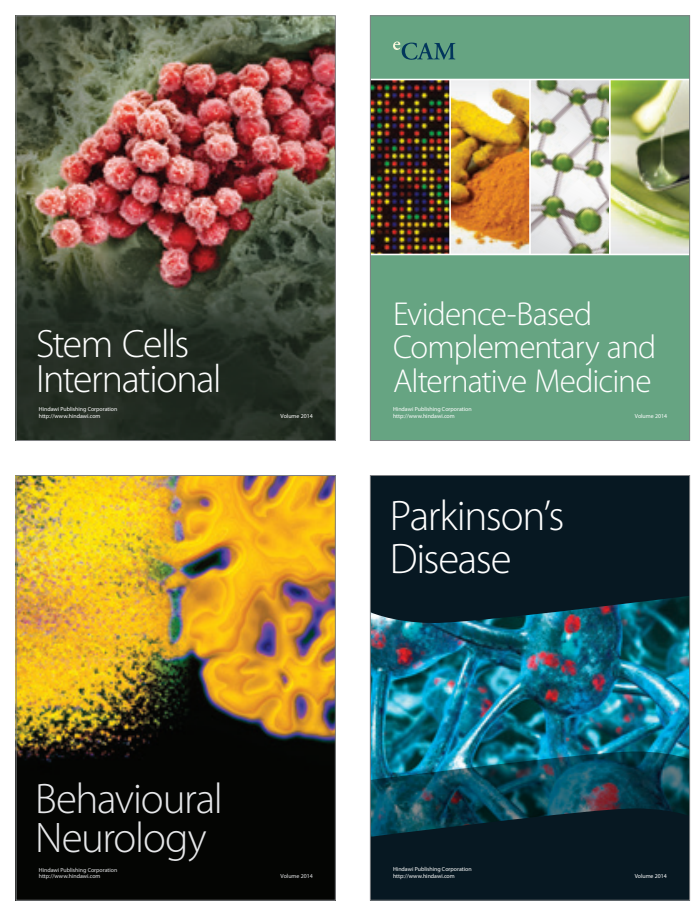

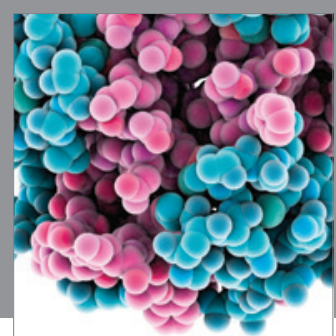

Journal of
Diabetes Research

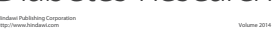

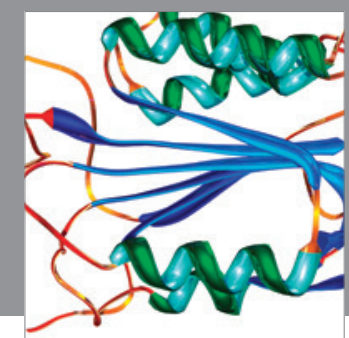

Disease Markers
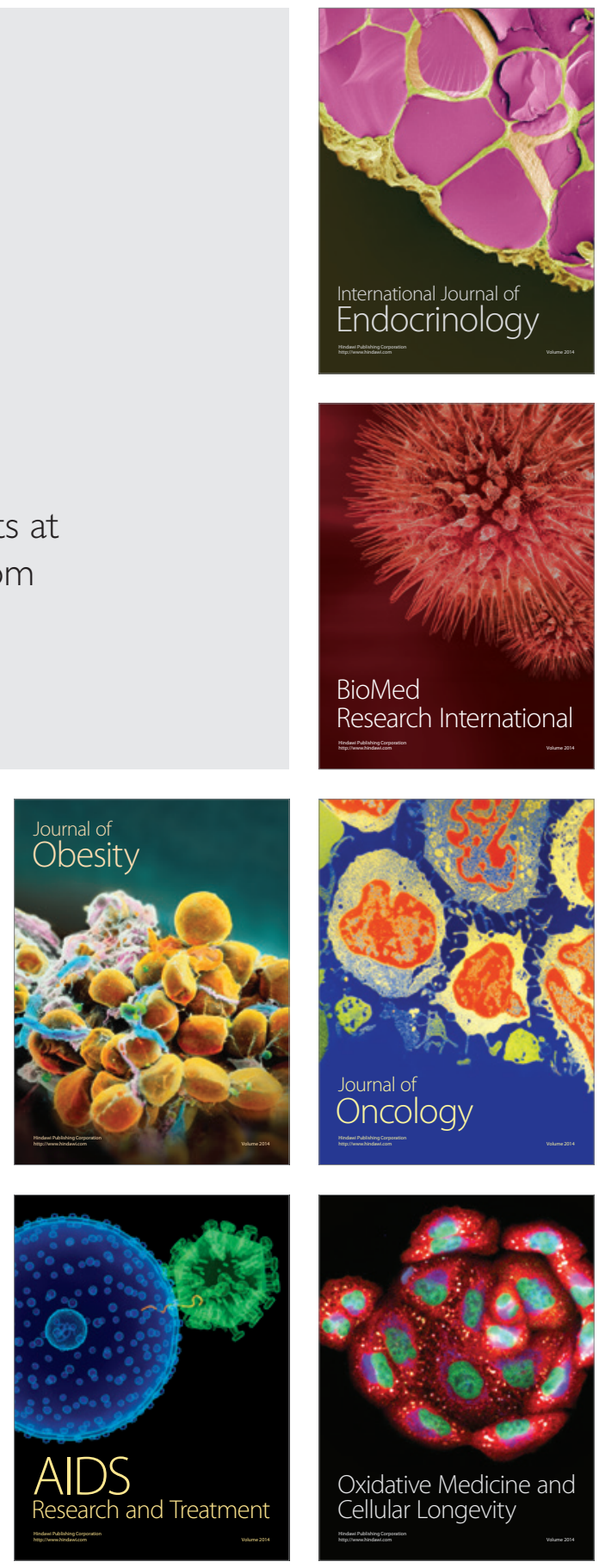